\title{
Electron-driven and thermal chemistry during water-assisted purification of platinum nanomaterials generated by electron beam induced deposition
}

\author{
Ziyan Warneke ${ }^{\ddagger 1}$, Markus Rohdenburg ${ }^{\ddagger 1}$, Jonas Warneke ${ }^{1,2}$, Janina Kopyra ${ }^{3}$ \\ and Petra Swiderek ${ }^{* 1}$
}

\section{Full Research Paper}

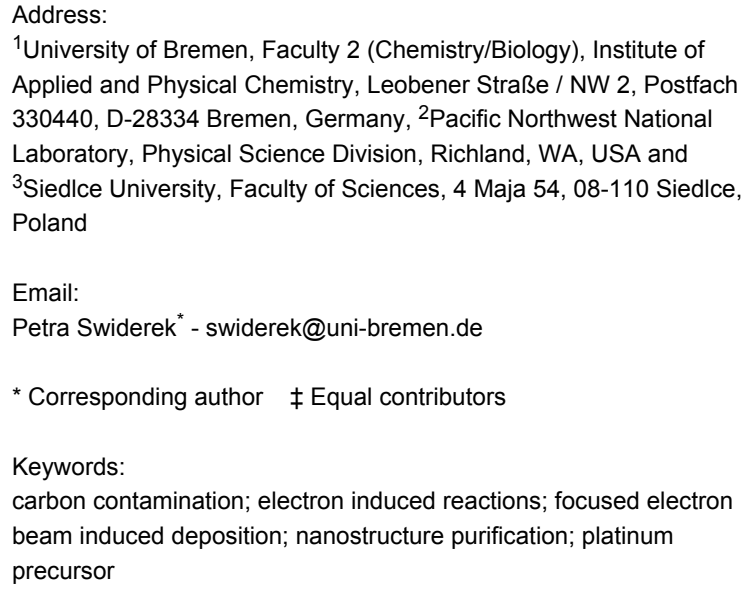

${ }^{1}$ University of Bremen, Faculty 2 (Chemistry/Biology), Institute of Applied and Physical Chemistry, Leobener Straße / NW 2, Postfach 330440, D-28334 Bremen, Germany, ${ }^{2}$ Pacific Northwest National Laboratory, Physical Science Division, Richland, WA, USA and ${ }^{3}$ Siedlce University, Faculty of Sciences, 4 Maja 54, 08-110 Siedlce, Poland

Email:

Petra Swiderek ${ }^{*}$ - swiderek@uni-bremen.de

* Corresponding author $\ddagger$ Equal contributors

Keywords:

carbon contamination; electron induced reactions; focused electron beam induced deposition; nanostructure purification; platinum precursor

Beilstein J. Nanotechnol. 2018, 9, 77-90. doi:10.3762/bjnano.9.10

Received: 07 August 2017

Accepted: 06 December 2017

Published: 08 January 2018

This article is part of the Thematic Series "Chemistry for electron-induced nanofabrication".

Associate Editor: J. J. Schneider

(C) 2018 Warneke et al.; licensee Beilstein-Institut.

License and terms: see end of document.

\begin{abstract}
Focused electron beam induced deposition (FEBID) is a versatile tool for the direct-write fabrication of nanostructures on surfaces. However, FEBID nanostructures are usually highly contaminated by carbon originating from the precursor used in the process. Recently, it was shown that platinum nanostructures produced by FEBID can be efficiently purified by electron irradiation in the presence of water. If such processes can be transferred to FEBID deposits produced from other carbon-containing precursors, a new general approach to the generation of pure metallic nanostructures could be implemented. Therefore this study aims to understand the chemical reactions that are fundamental to the water-assisted purification of platinum FEBID deposits generated from trimethyl(methylcyclopentadienyl)platinum(IV) $\left(\mathrm{MeCpPtMe}_{3}\right)$. The experiments performed under ultrahigh vacuum conditions apply a combination of different desorption experiments coupled with mass spectrometry to analyse reaction products. Electronstimulated desorption monitors species that leave the surface during electron exposure while post-irradiation thermal desorption spectrometry reveals products that evolve during subsequent thermal treatment. In addition, desorption of volatile products was also observed when a deposit produced by electron exposure was subsequently brought into contact with water. The results distinguish between contributions of thermal chemistry, direct chemistry between water and the deposit, and electron-induced reactions that all contribute to the purification process. We discuss reaction kinetics for the main volatile products $\mathrm{CO}$ and $\mathrm{CH}_{4}$ to obtain mechanistic information. The results provide novel insights into the chemistry that occurs during purification of FEBID nanostructures with implications also for the stability of the carbonaceous matrix of nanogranular FEBID materials under humid conditions.
\end{abstract}




\section{Introduction}

Focused electron beam induced deposition (FEBID) produces solid nanomaterials with size down to the sub-10 nm regime by decomposing precursor molecules adsorbed on a surface under a tightly focused high-energy electron beam [1]. Applications of this technology range from repair of masks for photolithography [2] and the fabrication of AFM tips [1] to novel photonic $[3,4]$ or plasmonically active [5] devices and sensor concepts [6]. Also, nanoscale structures grown by FEBID may possess promising magnetic properties $[7,8]$. However, metallic nanostructures produced by FEBID are often contaminated by considerable amounts of carbon, preventing them from fulfilling their desired functionality [1,9].

The main source of this impurity is the precursor itself that is used for the process. FEBID precursors typically contain atoms of the desired solid material and organic ligands that enhance their volatility. Metal organic precursors are thus used to fabricate metallic deposits. In the ideal case, a pure metal should remain at the surface while the organic ligands decompose into volatile products that are pumped away. However, this is usually not the case and material from the ligands tends to be incorporated in the deposit and thus deteriorates its physical properties [1,9-11].

Trimethyl(methylcyclopentadienyl)platinum(IV) $\left(\mathrm{MeCpPtMe}_{3}\right.$, Figure 1a) is widely applied as precursor for deposition of $\mathrm{Pt}$ because of its very favourable vapour pressure and good stability $[1,10]$. However, $\mathrm{MeCpPtMe}_{3}$ is a notoriously bad precursor for FEBID because it typically yields deposits with $\mathrm{Pt}$ content below $20 \%$ [1,9-11], which is insufficient for applications calling for pure metallic properties. In fact, deposits produced by FEBID from $\mathrm{MeCpPtMe}_{3}$ have a nanogranular structure, i.e., they consist of nanoscale Pt particles embedded in a carbonaceous matrix [12]. While FEBID-based PtC metalmatrix nanocomposites can serve, for instance, as transducing elements for humidity sensing [13], other applications call for high electrical conductivity. Therefore, different purification processes have been devised to turn the material into highpurity Pt [9,11,14-16]. In particular, post-deposition treatment with $\mathrm{O}_{2}$ at elevated temperature [9], the simultaneous exposure of the deposit to $\mathrm{O}_{2}$ and further electron irradiation [14-16], as well as FEBID of $\mathrm{MeCpPtMe}_{3}$ performed in the presence of $\mathrm{O}_{2}$ [15] have succeeded in yielding deposits with significantly improved Pt content [9] or even pure but often severely porous deposits [14-16].

It was recently shown that carbon layers in the presence of $\mathrm{H}_{2} \mathrm{O}$ vapour and graphene onto which a thin ice layer has been condensed can be efficiently etched by an electron beam [3,17]. Using a similar approach (Figure 1b), carbon-rich deposits pro-
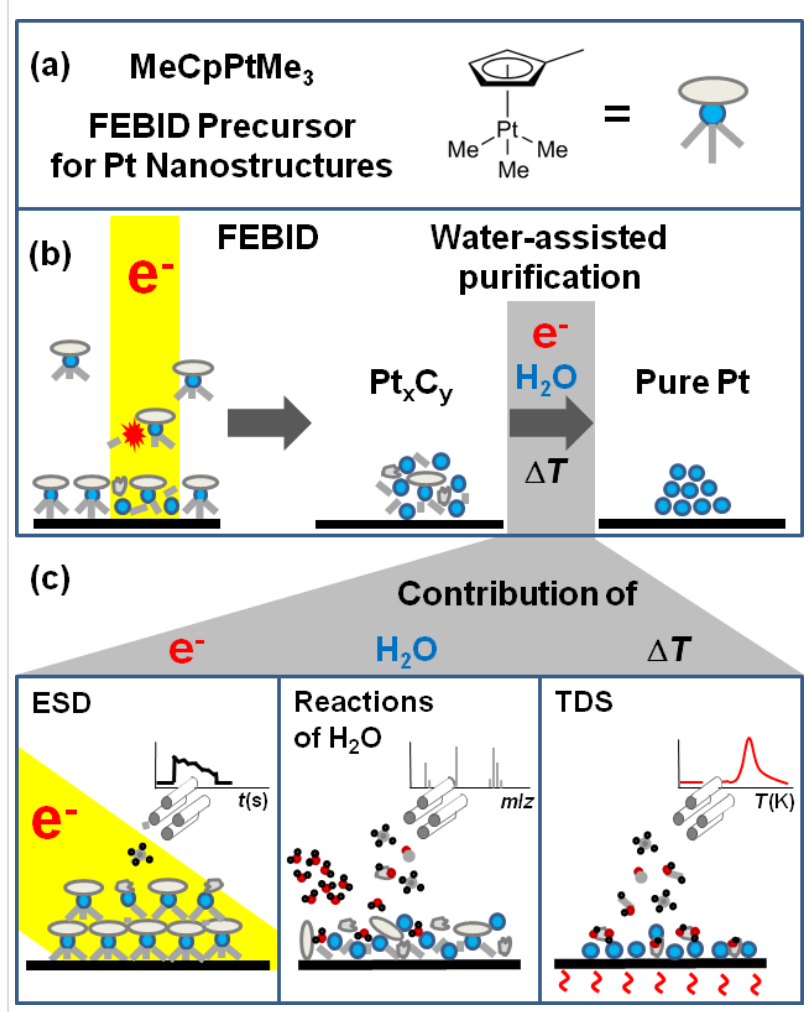

Figure 1: Molecular structure of the FEBID precursor trimethyl(methylcyclopentadienyl)platinum(IV) ( $\mathrm{MeCpPtMe}_{3}$ (a), schematic of the FEBID process and the subsequent water-assisted purification [12] of a deposit produced from $\mathrm{MeCpPtMe}_{3}$ (b), and mass spectrometric desorption experiments applied in this work to obtain insight in the underlying chemical reactions of the water-assisted purification process (c).

duced from the 'notoriously bad' precursor $\mathrm{MeCpPtMe}_{3}$ can be efficiently purified by a post-deposition electron-beam treatment in the presence of $\mathrm{H}_{2} \mathrm{O}$ vapour whereby a densely packed carbon- and oxygen-free Pt material is obtained [12]. $\mathrm{H}_{2} \mathrm{O}$ is a favourable purification reagent also because it is typically less aggressive towards electron gun filaments than $\mathrm{O}_{2}$. If this purification procedure can be fully understood, controlled, and transferred to FEBID deposits produced from other carbon-containing precursors, the portfolio of compounds suitable for FEBID would widen enormously. This would provide a new perspective to overcome the challenges that FEBID faces regarding the generation of pure metallic nanostructures. However, to reach this goal, a detailed understanding on the molecular level is required. The previous study [12] reported the purification process on a phenomenological level and did not provide insight in its underlying chemical reactions. It could only assume that these reactions involve the conversion of the initial carbon material to small volatile products $\left(\mathrm{CO}, \mathrm{CO}_{2}, \mathrm{CH}_{\mathrm{x}}\right)$ that, in the case of the FEBID deposit purification process, diffuse to the surface and desorb [12]. 
Here, we provide first insights on a molecular level into the underlying chemistry of this water-assisted purification process. We combine experiments on electron-stimulated desorption (ESD) and isothermal desorption of volatile products upon dosing of $\mathrm{H}_{2} \mathrm{O}$ with subsequent thermal desorption spectrometry (TDS). These experiments (Figure 1c) allow us to identify the volatile products, to distinguish between electron-driven reactions and contributions of thermal chemistry and to obtain insight into some mechanistic aspects of the water-assisted purification process. Our results provide a fundament for the molecular understanding and rational control of FEBID nanostructure purification using water as process gas [12] with additional implications for the stability of the carbonaceous matrix of nanogranular FEBID materials under humid conditions as relevant, for instance, in applications such as humidity sensing [13].

\section{Results and Discussion Thermal reactions between intact $\mathrm{MeCpPtMe}_{3}$ and $\mathrm{H}_{2} \mathrm{O}$}

In ALD processes for deposition of Pt performed at $100{ }^{\circ} \mathrm{C}$ and consisting of alternating cycles of precursor and $\mathrm{O}_{2}$ dosing, $\mathrm{MeCpPtMe}_{3}$ reacts with surface hydroxyl groups produced during a preceding $\mathrm{O}_{2}$ exposure half-cycle whereby $\mathrm{Pt}-\mathrm{O}$ bonds are formed and $\mathrm{CH}_{4}$ is released [18,19]. The analogous reaction between $\mathrm{MeCpPtMe}_{3}$ and $\mathrm{H}_{2} \mathrm{O}$ does not contribute noticeably in our low temperature $(105 \mathrm{~K})$ experiments as verified by exposing a condensed layer of the precursor to $\mathrm{H}_{2} \mathrm{O}$ vapour. During leaking of $\mathrm{H}_{2} \mathrm{O}$ (Figure 2a), generation of $\mathrm{CH}_{4}$ was indeed not seen. In a subsequent TDS experiment (Figure 2b), the desorption of $\mathrm{H}_{2} \mathrm{O}$ and intact $\mathrm{MeCpPtMe}_{3}$ (compare Supporting Information File 1, Figure S1) was observed at $150 \mathrm{~K}$ and $210 \mathrm{~K}$, respectively. We note that at temperatures between $250 \mathrm{~K}$ and $350 \mathrm{~K}$ slightly increased intensities of the methane signals were detected. These amounts are, however, negligible in comparison to reaction products formed under electron irradiation, as shown in the following.

\section{Electron-induced degradation of multilayer condensed films of $\mathrm{MeCpPtMe}_{3}$}

Electron-stimulated desorption was first measured from multilayer condensed films of $\mathrm{MeCpPtMe}_{3}$ without added $\mathrm{H}_{2} \mathrm{O}$ as reference for subsequent experiments (Figure 3). In accord with previous results [10], material is removed from the layer exclusively in form of $\mathrm{CH}_{4}$ molecules. This is obvious from the intensity ratio of 1:0.9 of the MS signals recorded at $\mathrm{m} / \mathrm{z} 16$ and 15 which is characteristic of $\mathrm{CH}_{4}$ thus excluding noticeable contributions of $\mathrm{CH}_{3}$ radicals that would lead to additional intensity of the $m / z 15$ signal. It was proposed previously that the exclusive observation of $\mathrm{CH}_{4}$ can be explained by reaction of desorbing $\mathrm{CH}_{3}$ radicals with hydrogen from the walls of the

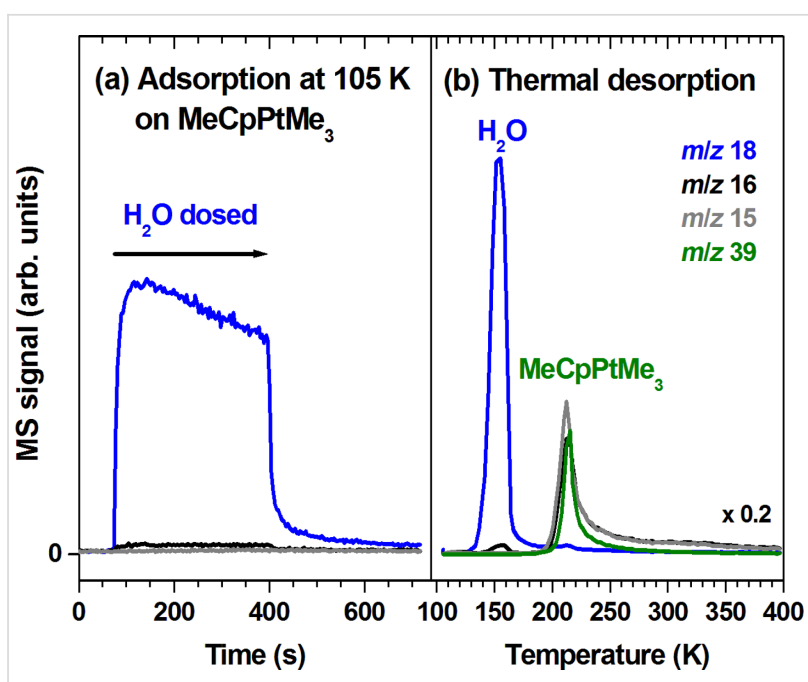

Figure 2: (a) MS signals recorded during leaking of $\mathrm{H}_{2} \mathrm{O}$ onto a film of $\mathrm{MeCpPtMe}_{3}$ with thickness corresponding to 30 monolayers deposited on a Ta substrate held at $105 \mathrm{~K}$. The amount of $\mathrm{H}_{2} \mathrm{O}$ vapour applied in this experiment was the same as that used for depositing the $\mathrm{MeCpPtMe}_{3}$ multilayer film. The $\mathrm{m} / \mathrm{z}$ ratios 18,16 , and 15 were recorded to monitor leaking of $\mathrm{H}_{2} \mathrm{O}$ as well as possible formation of $\mathrm{CH}_{4}$. For $\mathrm{CH}_{4}$, the relative intensity of the $\mathrm{m} / \mathrm{z}$ ratios 16 and 15 amounts to 1:0.9 [10]. (b) TDS experiment performed subsequently on the same sample. Here, $\mathrm{m} / \mathrm{z} 39\left(\mathrm{C}_{3} \mathrm{H}_{3}{ }^{+}\right)$is also included as a characteristic MS signal of $\mathrm{MeCpPtMe}_{3}$ (see [11]).

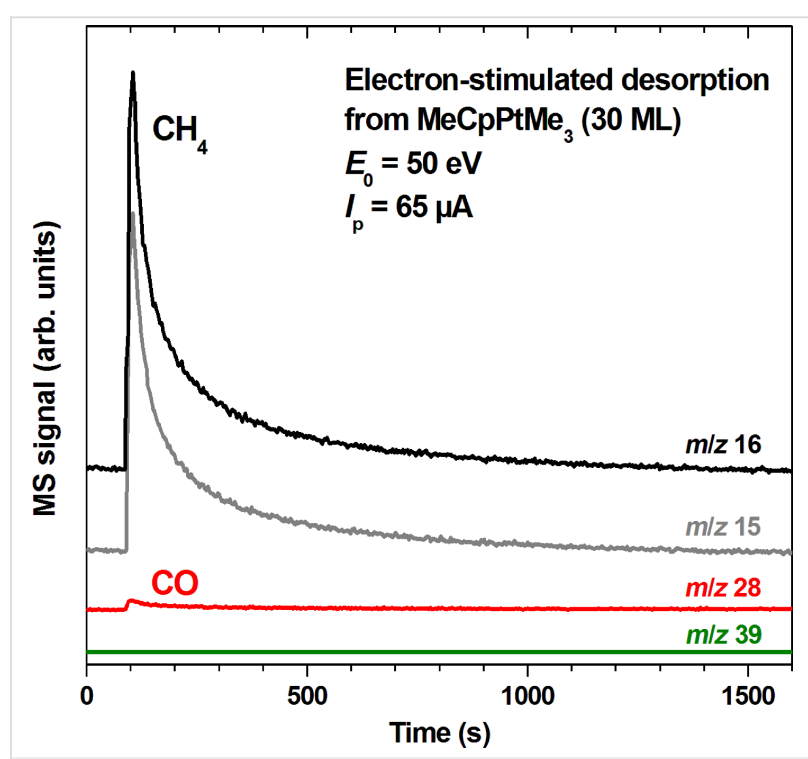

Figure 3: Electron-stimulated desorption from a 30 layer film of $\mathrm{MeCpPtMe}_{3}$ on a Ta substrate held at $105 \mathrm{~K}$. An incident electron current $I_{\mathrm{p}}$ of $13 \mu \mathrm{A} / \mathrm{cm}^{2}$ was applied at $E_{0}=50 \mathrm{eV}$. The $\mathrm{m} / \mathrm{z}$ ratios 16 and 15 were recorded to monitor formation of $\mathrm{CH}_{4}$ for which the relative intensity of the $\mathrm{m} / \mathrm{z}$ ratios 16 and 15 amounts to $1: 0.9$ [10]. The $\mathrm{m} / \mathrm{z}$ ratio 28 indicates desorption of $\mathrm{CO}$ and of traces of ethane (see Supporting Information File 1, Figure S2), while $\mathrm{m} / \mathrm{z} 39\left(\mathrm{C}_{3} \mathrm{H}_{3}{ }^{+}\right)$is also included as a characteristic MS signal of $\mathrm{MeCpPtMe}_{3}$ (see [11]).

UHV chamber that occur before $\mathrm{CH}_{3}$ can reach the mass spectrometer [11]. Based on previous results from our setup, we can exclude such an artifact. In fact, ESD of $\mathrm{CH}_{3}$ was clearly ob- 
served from condensed layers of acetone and acetylacetone [20] in the same setup used also for the experiments described here.

Beside the predominant $\mathrm{CH}_{4}$, a small desorption signal with $\mathrm{m} / \mathrm{z}$ ratio 28 is seen at the start of irradiation (Figure 3). It was identified as a minor $\mathrm{CO}$ background signal with equally small contributions of ethane $\left(\mathrm{C}_{2} \mathrm{H}_{6}\right)$ according to a mass spectrum recorded during the initial stages of electron exposure (Supporting Information File 1, Figure S2). The very small quantity of $\mathrm{C}_{2} \mathrm{H}_{6}$, confirms again the previous conclusion [10] that recombination of $\mathrm{CH}_{3}$ ligands dissociating from the precursor is not a relevant reaction in the electron-induced degradation of $\mathrm{MeCpPtMe}_{3}$ layers. In addition, the $m / z 39$ trace gives evidence that ESD of the precursor is negligible.

According to previous gas phase experiments [21], the dominant fragmentation proceeding via dissociative electron attachment (DEA) leads to loss of only one neutral $\mathrm{CH}_{3}$ ligand. DEA occurs at low electron energies characteristic of the secondary electrons that are released in large numbers under impact of a high energy primary electron beam and are therefore thought to make major contributions to deposit formation. Assuming that the ligand material remaining behind after loss of the first $\mathrm{CH}_{3}$ is embedded in the deposit, DEA thus explains the deposit composition of $\mathrm{Pt} / \mathrm{C}=1: 8$ that was obtained when the precursor was decomposed at $180 \mathrm{~K}$ by electron exposure in UHV, i.e., without contributions of residual vapours to deposit formation [10]. From this perspective [11], it is surprising that only $\mathrm{CH}_{4}$ desorbs during electron irradiation and not $\mathrm{CH}_{3}$ itself. The previous ESD data on $\mathrm{MeCpPtMe}_{3}$ [10] revealed a linear increase of the $\mathrm{CH}_{4}$ yield as function of coverage. This was ascribed to an intramolecular reaction as origin of $\mathrm{CH}_{4}$ which is supported by the very small amount of $\mathrm{C}_{2} \mathrm{H}_{6}$ production as also observed here. The latter would be expected to result from recombination of $\mathrm{CH}_{3}$ radicals that have dissociated from the precursor. When electron irradiation is performed at $E_{0}$ well above the ionization threshold, as in the present and previous [10] surface studies, precursor fragmentation is driven by electron impact ionization (EI). In this regime, loss of $\mathrm{CH}_{4}$ from the precursors has in fact been observed in gas phase mass spectrometry $[11,20]$. Nonetheless, the abundances of specific fragment ions observed in the positive ion mass spectra suggest that fragmentation does not exclusively produce $\mathrm{CH}_{4}$ but also some $\mathrm{CH}_{3}$ [21]. ESD of $\mathrm{CH}_{3}$ from a condensed layer of $\mathrm{MeCpPtMe}_{3}$ during electron exposure would thus again be anticipated.

Considering these electron-induced dissociation reactions of $\mathrm{MeCpPtMe}_{3}$, trapping of fragments in the precursor layer may provide a more convincing explanation for the lack of $\mathrm{CH}_{3}$ in ESD. Such an effect was observed previously in the case of acetylacetone where gas-phase experiments demonstrated that electron-induced loss of $\mathrm{CH}_{3}$ is significant in the monomers but is completely suppressed in the dimer and ESD of $\mathrm{CH}_{3}$ from a condensed layer of the compound is also weak [21]. In the case of $\mathrm{MeCpPtMe}_{3}$, the $\mathrm{CH}_{3}$ radical may add efficiently to the unsaturated cyclopentadienyl ligand or abstract a hydrogen atom from a second ligand. In the second case, abstraction from a $\mathrm{CH}_{3}$ ligand would yield an intermediate with $\mathrm{Pt}=\mathrm{CH}_{2}$ unit, i.e., a carbene ligand. Such intermediates are akin to species occurring in dehydrogenation reactions of $\mathrm{CH}_{3}$ groups on $\mathrm{Pt}$ surfaces [22] and have also been observed in the gas phase following reactive interaction between $\mathrm{Pt}^{+}$ions and $\mathrm{CH}_{4}$ [23].

Unfortunately, absolute cross section data for DEA and EI of $\mathrm{MeCpPtMe}_{3}$ as well as a detailed understanding of the fragmentation mechanisms following EI do not exist to the best of our knowledge. Furthermore, neutral dissociation (ND), a non-resonant process with a threshold comparable to EI and leading to the formation of uncharged fragments, may also play a role in the observed $\mathrm{CH}_{4}$ production. However, data on ND are generally difficult to obtain and do not exist so far for $\mathrm{MeCpPtMe}_{3}$. A complete set of such cross section data supplemented by theoretical studies of the electron-induced dissociation and of subsequent reactions of the resulting fragments would help to better understand which of the initiating electron-precursor interactions is more likely to dominate deposit formation.

\section{Interplay of electron-induced and thermal degradation of $\mathrm{MeCpPtMe}_{3}$}

As shown in Figure 2 and Supporting Information File 1, Figure $\mathrm{S} 1$ and in accord with previous results [10], non-irradiated $\mathrm{MeCpPtMe}_{3}$ desorbs in UHV without noticeable thermal decomposition. To investigate the contribution of thermal decomposition to the FEBID process, a TDS experiment was performed after extensive irradiation, i.e., at a time when the ESD signals of $\mathrm{CH}_{4}$ had decayed approximately to the baseline level (Figure 4). As shown previously [10], changes in oxidation state of Pt, loss of carbon, and the complete loss of the infrared $\mathrm{CH}$ stretching bands proceed on the same time scale as the decay of $\mathrm{CH}_{4}$ signals in ESD. Therefore, the complete decay of $\mathrm{CH}_{4}$ desorption appears to indicate that the precursor layer has been fully converted to a deposit as also obtained in a FEBID process. We note that such fully electron-degraded layers were also used to study the reactions underlying the water-assisted purification process (see following sections).

Figure 4 reveals that thermal reactions in fact occur in the degraded precursor layers. In the case of the irradiated sample (Figure 4, bottom), a small desorption peak near $220 \mathrm{~K}$ for $\mathrm{m} / \mathrm{z}$ 39 and 28 suggests that a certain amount of precursor or a chemically very similar and equally volatile species was left on the substrate, despite the fact that ESD of $\mathrm{CH}_{4}$ had ceased. 


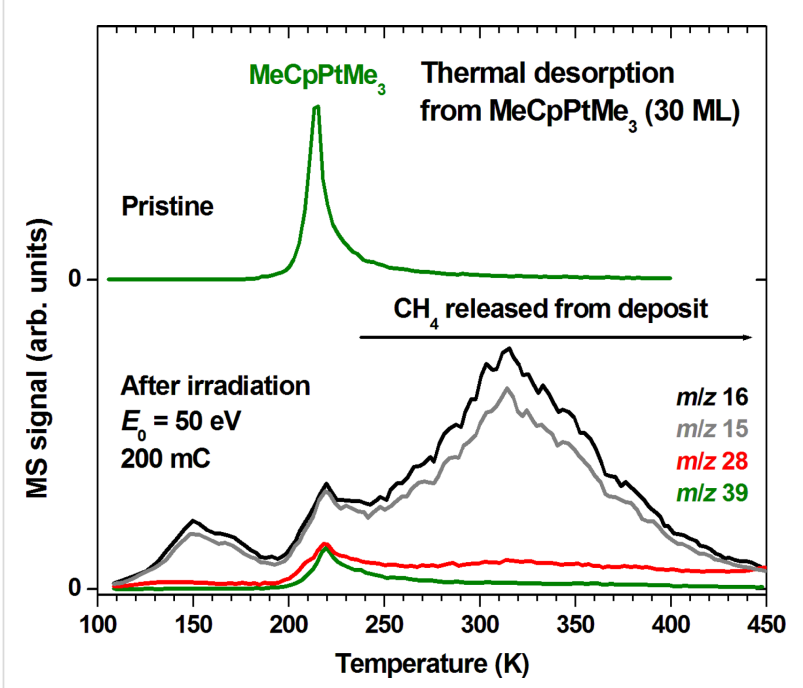

Figure 4: Thermal desorption spectra recorded from a 30 layer film of $\mathrm{MeCpPtMe}_{3}$ as deposited at $105 \mathrm{~K}$ on a Ta substrate (top) and from the $\mathrm{MeCpPtMe}_{3}$ film prepared for the experiment shown in Figure 3 but after a total electron exposure of $40 \mathrm{mC} / \mathrm{cm}^{2}$ at $E_{0}=50 \mathrm{eV}$ (bottom). The $\mathrm{m} / \mathrm{z}$ ratios 16 , and 15 were recorded to monitor the possible formation of $\mathrm{CH}_{4}$ for which their relative intensities amount to 1:0.9 [10]. The $\mathrm{m} / \mathrm{z}$ ratio 28 is included to monitor possible release of $\mathrm{C}_{2} \mathrm{H}_{6}$ and $\mathrm{m} / \mathrm{z} 39\left(\mathrm{C}_{3} \mathrm{H}_{3}{ }^{+}\right)$is included as a characteristic MS signal of $\mathrm{MeCpPtMe}_{3}$ (see [11]). Only $\mathrm{m} / \mathrm{z} 39$ is shown for clarity in the case of the fresh layer (top) where other desorption signals were absent (see Supporting Information File 1, Figure S1).

Attenuation of the electron beam in the relatively thick $\mathrm{MeCpPtMe}_{3}$ layer used in the present experiment may have led to incomplete conversion of deeper layers and can thus explain the remaining precursor signal. More important, however, is the thermal desorption of additional $\mathrm{CH}_{4}$ over a wide temperature range as evident from the TDS curves recorded at $\mathrm{m} / \mathrm{z} 15$ and 16. These desorption signals were absent in non-irradiated layers (Supporting Information File 1, Figure S1). The desorption signals around $150 \mathrm{~K}$ and at the desorption temperature of $\mathrm{MeCpPtMe}_{3}$ can be explained by release of some $\mathrm{CH}_{4}$ that was retained in the thick layer at $105 \mathrm{~K}$. In contrast, the release of $\mathrm{CH}_{4}$ above $230 \mathrm{~K}$ points to thermal decomposition of less volatile products formed during electron irradiation of $\mathrm{MeCpPtMe}_{3}$. After desorption of the precursor, these products remain on the surface and undergo thermal reactions as the temperature is further increased. The signal $\mathrm{m} / \mathrm{z} 28$ observed in the same temperature range and reaching up to $450 \mathrm{~K}$ points to the formation of $\mathrm{C}_{2} \mathrm{H}_{6}$ or $\mathrm{C}_{2} \mathrm{H}_{4}$ as a side product of this thermal decay. This result gives evidence that decomposition of the precursor is not only an electron-driven process but also proceeds via additional thermal chemistry.

Such thermal chemistry that assists the removal of ligands from the precursor has been described before. In particular, previous surface science studies on the FEBID precursor $\mathrm{Pt}\left(\mathrm{PF}_{3}\right)_{4}$ have shown that the initial electron-induced fragmentation leads to loss of one intact $\mathrm{PF}_{3}$ ligand while further irradiation merely removes additional fluorine [24]. In contrast, more intact ligands are removed by increasing the temperature resulting in a deposit with higher Pt content [25]. Here we show that the contributions of electron-induced and thermal reactions to precursor decomposition can also be deduced from ESD and TDS experiments. From the MS intensities during ESD (Figure 3) and post-irradiation TDS (Figure 4) we can estimate that in the case of $\mathrm{MeCpPtMe}_{3}$ roughly 1.5 times more $\mathrm{CH}_{4}$ desorbs as a consequence of thermal processes than during the previous irradiation at $105 \mathrm{~K}$. About half of this thermal $\mathrm{CH}_{4}$ release has occurred up to room temperature alone thus making a significant contribution to deposit formation. In addition, the further thermal decomposition extending up to $450 \mathrm{~K}$ is relevant to purification by post-deposition thermal purification processes. This result shows that a combination of ESD and TDS can provide an estimate of the relative contribution of electron-induced and thermal reactions in a given FEBID process.

\section{Water- and electron-induced reactions in electron-degraded multilayers of $\mathrm{MeCpPtMe}_{3}$}

The fact that FEBID deposits produced from $\mathrm{MeCpPtMe}_{3}$ can be purified by electron irradiation in the presence of $\mathrm{H}_{2} \mathrm{O}$ vapour [12] implies that as a consequence of electron exposure the deposit reacts readily with $\mathrm{H}_{2} \mathrm{O}$. To resolve under which conditions this reactivity sets in, thin condensed layers of $\mathrm{MeCpPtMe}_{3}$ were degraded by electron exposure to serve as model for a FEBID deposit. Specific amounts of $\mathrm{H}_{2} \mathrm{O}$ were then dosed onto the electron-degraded precursor layers held at $110 \mathrm{~K}$. At this temperature, $\mathrm{H}_{2} \mathrm{O}$ condenses on the substrate. Reactions were monitored by measuring MS signals of neutral products that desorbed from the surface during dosing of $\mathrm{H}_{2} \mathrm{O}$ and during subsequent or simultaneous electron exposure. Also, the effect of annealing was investigated.

In a first experiment (Figure 5), a 30 monolayer film of $\mathrm{MeCpPtMe}_{3}$ was partially degraded by electron irradiation until ESD of $\mathrm{CH}_{4}$ had decreased to about $50 \%$ of its initial value. At this stage of decomposition, a significant percentage of the precursor molecules must have lost at least one of their methyl groups. We note that the same products desorb as seen in Figure 3 despite the lower electron energy applied here. This is in accord with the result of a previous study on the electron-induced decomposition of $\mathrm{MeCpPtMe}_{3}$ by high-resolution electron energy loss spectroscopy (HREELS) [26] which has revealed that the nature of the deposit is similar for $E_{0}=50 \mathrm{eV}$ and $500 \mathrm{eV} \cdot \mathrm{H}_{2} \mathrm{O}$ was dosed onto this partially degraded precursor layer without prior annealing. This led to desorption of only small amounts of $\mathrm{CH}_{4}$ (Figure 5, between 400 and $600 \mathrm{~s}$ ) as evident from a slight increase of the $m / z 15$ signal. This indi- 


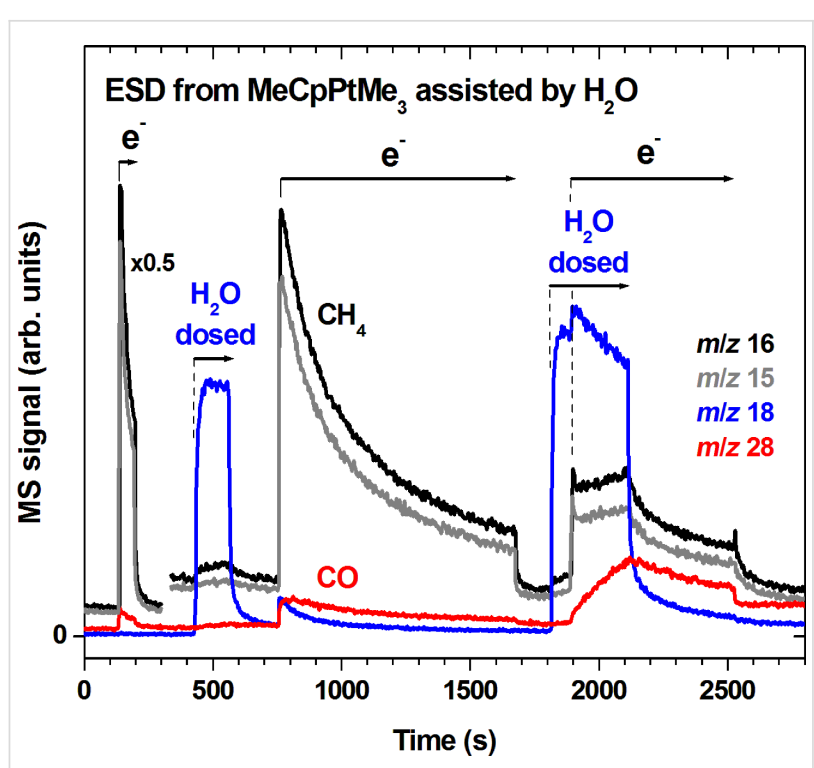

Figure 5: MS signals recorded during repeated electron exposures $\left(\mathrm{e}^{-}\right)$and leaking of $\mathrm{H}_{2} \mathrm{O}\left(\mathrm{H}_{2} \mathrm{O}\right.$ dosed) onto a 30 layer film of $\mathrm{MeCpPtMe}_{3}$ deposited and held at $105 \mathrm{~K}$. During the first $\mathrm{H}_{2} \mathrm{O}$ leaking, the amount of vapour was $50 \%$ of that used for depositing the $\mathrm{MeCpPtMe}_{3}$ multilayer film. For the second leaking, the amount of $\mathrm{H}_{2} \mathrm{O}$ vapour was increased by a factor of two. During the three electron irradiation periods, exposures of $1.04 \mathrm{mC} / \mathrm{cm}^{2}$ (start at $130 \mathrm{~s}$, $I_{\mathrm{p}}=17 \mu \mathrm{A} / \mathrm{cm}^{2}$ ), $20 \mathrm{mC} / \mathrm{cm}^{2}$ (start at $750 \mathrm{~s}, I_{\mathrm{p}}=22 \mu \mathrm{A} / \mathrm{cm}^{2}$ ), and $14 \mathrm{mC} / \mathrm{cm}^{2}$ (start at $1900 \mathrm{~s}, I_{\mathrm{p}}=22 \mu \mathrm{A} / \mathrm{cm}^{2}$ ) were applied at $E_{0}=31 \mathrm{eV}$. A lower $E_{0}$ as compared to Figure 3 was applied here to degrade the layers more slowly and consequently achieve better control over the number of injected electrons. The $\mathrm{m} / \mathrm{z}$ ratios 18,16 , 15 , and 28 were recorded to monitor leaking of $\mathrm{H}_{2} \mathrm{O}$ as well as possible formation of $\mathrm{CH}_{4}$ (relative intensity of $\mathrm{m} / \mathrm{z}$ ratios 16 and 15 amounts to $1: 0.9$ [10]) and $\mathrm{CO}$. Note that $\mathrm{m} / \mathrm{z} 16$ also contains a minor fragment signal of $\mathrm{H}_{2} \mathrm{O}$.

cates that precursor fragments can be hydrolyzed to some extent but this reaction is much less efficient for removal of organic material than the initial electron irradiation. When electron exposure was resumed (at $750 \mathrm{~s}$ ), the ESD rate of $\mathrm{CH}_{4}$ jumped back to its previous value at the end of the first irradiation period indicating that no major changes had incurred to the precursor layer during dosing of $\mathrm{H}_{2} \mathrm{O}$. Also, as $\mathrm{H}_{2} \mathrm{O}$ does not hinder desorption of $\mathrm{CH}_{4}$, it must either have diffused into the precursor layer or grown on top in porous form. The presence of $\mathrm{H}_{2} \mathrm{O}$ in the layer is obvious from an ESD signal at $\mathrm{m} / \mathrm{z} 18$ with moderate intensity that set in immediately with the start of irradiation and decayed afterwards. However, irradiation now also led to ESD of CO (see also Supporting Information File 1, Figure S3) with slightly delayed onset and a much slower decay as compared to $\mathrm{H}_{2} \mathrm{O}$ and $\mathrm{CH}_{4}$. Comparative blind experiments on ESD from the substrate without precursor layer (not shown) revealed a small background desorption signal in the $\mathrm{m} / \mathrm{z} 28$ curve akin to that seen during the initial electron irradiation in Figure 5. However, the characteristically slower decay of this signal during the second electron exposure in Figure 5 implies that this $\mathrm{CO}$ desorption is not a background effect but results from a reaction in the precursor layer during electron exposure in the presence of $\mathrm{H}_{2} \mathrm{O}$.

Electron irradiation was switched off again when ESD of $\mathrm{CH}_{4}$ had decreased to about $10 \%$ of its initial value (at $1700 \mathrm{~s}$ ). Again, dosing of $\mathrm{H}_{2} \mathrm{O}$ alone (starting at $1800 \mathrm{~s}$ ) led to only a small desorption signal of $\mathrm{CH}_{4}$. In contrast, switching on electron exposure (at $1900 \mathrm{~s}$ ) during dosing of $\mathrm{H}_{2} \mathrm{O}$ initiated an immediate onset and subsequent further increase in the ESD rate of $\mathrm{CH}_{4}$ and a continuous increase of the $\mathrm{CO}$ ESD rate while condensation of $\mathrm{H}_{2} \mathrm{O}$ proceeded. Here, the amounts of $\mathrm{CO}$ clearly exceeded the background levels observed in the blind experiment. The increase of the $\mathrm{CH}_{4}$ ESD rate with continuing dosing of $\mathrm{H}_{2} \mathrm{O}$ during the third irradiation period implies that $\mathrm{H}_{2} \mathrm{O}$ is a limiting factor in this reaction. When the supply of $\mathrm{H}_{2} \mathrm{O}$ was stopped again (at $2100 \mathrm{~s}$ ), all MS signals leveled off slowly and more strongly after electron exposure was also stopped (at $2500 \mathrm{~s}$ ).

ESD signals of $\mathrm{CO}$ during dosing of $\mathrm{H}_{2} \mathrm{O}$ but before the start of electron irradiation are too small to be visible in Figure 5. Therefore, the evolution of the $\mathrm{CO}$ signal has been investigated in a second set of experiments in which $\mathrm{H}_{2} \mathrm{O}$ was dosed only after ESD of $\mathrm{CH}_{4}$ had decayed nearly back to the background signal and irradiation was performed only after dosing of $\mathrm{H}_{2} \mathrm{O}$ (Figure 6). In fact, Figure 6 shows a small and continuous increase of the MS signal at $\mathrm{m} / z 28$ during dosing of $\mathrm{H}_{2} \mathrm{O}$. When electron irradiation was started after leaking of $\mathrm{H}_{2} \mathrm{O}$ without prior annealing, the desorption rate of $\mathrm{CO}$ increased strongly revealing again the action of $\mathrm{H}_{2} \mathrm{O}$ in contact with the deposit (Figure 6a). Careful inspection of the ESD signal reveals that the release of $\mathrm{CO}$ is somewhat delayed as compared to ESD of $\mathrm{H}_{2} \mathrm{O}$ implying that $\mathrm{CO}$ is formed by an intermediate that is then decomposed by electron exposure. We note that some minor production of $\mathrm{CO}_{2}$ was also observed and that a similar result was obtained in experiments performed in the same way but at $19 \mathrm{eV}$ (not shown). As a difference, ESD of $\mathrm{H}_{2} \mathrm{O}$ was very small at $19 \mathrm{eV}$.

The experiments summarized in Figure 5 and Figure 6 show that the combined action of $\mathrm{H}_{2} \mathrm{O}$ and electron exposure is required for an efficient removal of organic material at cryogenic temperature. In fact, as seen in Figure 5, desorption of products in the presence of $\mathrm{H}_{2} \mathrm{O}$ alone proceeds at a relatively low rate even after a large fraction of $\mathrm{MeCpPtMe}_{3}$ has been decomposed by electron irradiation. This indicates that the intermediates that reside in the deposit after the initial electron-induced fragmentation of the precursor are not very reactive towards $\mathrm{H}_{2} \mathrm{O}$ at cryogenic temperature. The reason is difficult to trace without detailed knowledge of the structure of these species. However, it has been shown that $\mathrm{H}_{2} \mathrm{O}$ reacts only slowly with a 

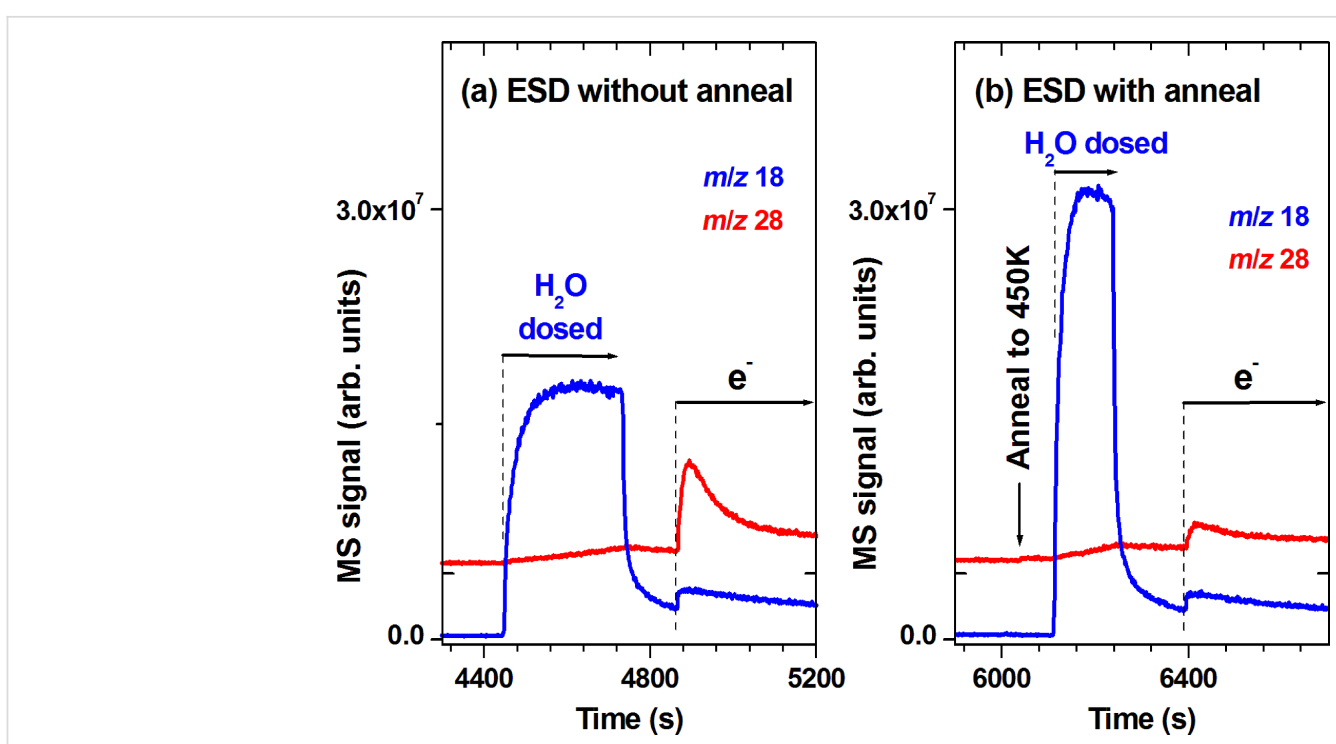

Figure 6: MS signals recorded during leaking of $\mathrm{H}_{2} \mathrm{O}\left(\mathrm{H}_{2} \mathrm{O}\right)$ and electron exposure at $E_{0}=31 \mathrm{eV}\left(\mathrm{e}^{-}\right)$of a 30 layer film of MeCpPtMe $e_{3}$ deposited and held at $105 \mathrm{~K}$. Prior to the processes steps shown here, the film was degraded by electron exposure at the same energy until $\mathrm{ESD}_{\mathrm{S}} \mathrm{CH}_{4}$ had decayed. The amount of $\mathrm{H}_{2} \mathrm{O}$ vapour was the same as used for depositing the $\mathrm{MeCpPtMe}_{3}$ film. This procedure was performed (a) without and (b) with an annealing step to $450 \mathrm{~K}$ applied prior to leaking of $\mathrm{H}_{2} \mathrm{O}$. The $\mathrm{m} / \mathrm{z}$ ratios 18 and 28 are shown to visualize leaking of $\mathrm{H}_{2} \mathrm{O}$ and formation of CO. The dashed line marks the start of the irradiation period with (a) $I_{\mathrm{p}}=10 \mu \mathrm{A} / \mathrm{cm}^{2}$ and (b) $I_{\mathrm{p}}=12 \mu \mathrm{A} / \mathrm{cm}^{2}$.

model complex $\mathrm{PtCH}_{2}{ }^{+}$prepared in gas phase [23] suggesting that such reactive intermediates may be relatively stable.

Electron-initiated reactions of $\mathrm{H}_{2} \mathrm{O}$ itself are a conceivable reason for the enhanced precursor or deposit degradation seen in Figure 5 and Figure 6 when the electron beam is switched on in the presence of $\mathrm{H}_{2} \mathrm{O}$. Electron exposure of condensed $\mathrm{H}_{2} \mathrm{O}$ and clusters of $\mathrm{H}_{2} \mathrm{O}$ at $E_{0}$ above the ionization threshold is known to induce the following proton transfer [27]

$$
\mathrm{H}_{2} \mathrm{O}^{+}+\mathrm{H}_{2} \mathrm{O} \rightarrow \mathrm{H}_{3} \mathrm{O}^{+}+\cdot \mathrm{OH}
$$

and thereby yields $\mathrm{OH}$ radicals. As $\mathrm{MeCpPtMe}_{3}$ tends to lose its cyclopentadienyl ligand when exposed to acidic conditions $[28,29]$, a similar process is conceivable following ionizationinduced proton transfer from $\mathrm{H}_{2} \mathrm{O}$ to the precursor

$$
\mathrm{H}_{2} \mathrm{O}^{+}+\mathrm{MeCpPtMe}_{3} \rightarrow \mathrm{MeCpH}+\mathrm{PtMe}_{3}{ }^{+}+\cdot \mathrm{OH} .
$$

This would weaken the bonding between the negatively charged $\mathrm{MeCp}$ ligand and $\mathrm{Pt}$ thus exposing the latter to attack by $\mathrm{H}_{2} \mathrm{O}$ or the more reactive $\mathrm{OH}$ radical. This paves the avenues to oxidation of the ligands which is obvious here through formation of $\mathrm{CO}$ and even $\mathrm{CO}_{2}$. Such a scenario is supported by the fact that $\mathrm{Pt}$ complexes in aqueous solution are catalysts for oxidation of alkanes and other organic compounds [30].

Electron-induced reactions involving $\mathrm{OH}$ radicals have recently been discussed in relation to the electron-induced formation of ethanol in condensed mixtures of ethylene and $\mathrm{H}_{2} \mathrm{O}$ [31]. In this study, it was observed that product formation via reactions that are held to be maintained by a chain reaction involving $\mathrm{OH}$ radicals became only noticeable when the irradiated molecular layers were sufficiently thick. This effect was explained by deactivation of the $\mathrm{OH}$ radicals by capturing of thermalized electrons, an effect that can only occur near the surface and not below a depth roughly corresponding to the effective penetration depth of the electron beam. Purification of actual FEBID deposits was also observed to be more efficient at a greater depth within the layer [12] supporting that a contribution of $\mathrm{OH}$ radicals is a reasonable scenario in the context of deposit purification by electron exposure in the presence of $\mathrm{H}_{2} \mathrm{O}$.

When the sample was annealed by heating to $450 \mathrm{~K}$ prior to dosing of $\mathrm{H}_{2} \mathrm{O}$, significantly less $\mathrm{CO}$ desorbed during subsequent electron exposure (Figure 6b). This can be traced back to loss of material during the annealing as seen in TDS data (Figure 7). As in Figure 4, additional thermal decomposition is witnessed by desorption of further organic material $(\mathrm{m} / \mathrm{z} 15)$ around the desorption temperatures of $\mathrm{H}_{2} \mathrm{O}(150 \mathrm{~K})$ and remaining precursor $(210 \mathrm{~K})$ as well as, more strongly, above $220 \mathrm{~K}$ with maximum rate around room temperature. Desorption has again mostly ceased at $450 \mathrm{~K}$ in line with the earlier result showing that complete reduction of $\mathrm{MeCpPtMe}_{3}$ in CVD processes assisted by $\mathrm{H}_{2}$ can be achieved just below $400 \mathrm{~K}$ [19]. We conclude that too little material is left at the surface to gain deeper insight in the purification process when only a single multilayer film of $\mathrm{MeCpPtMe}_{3}$ has been decomposed. 


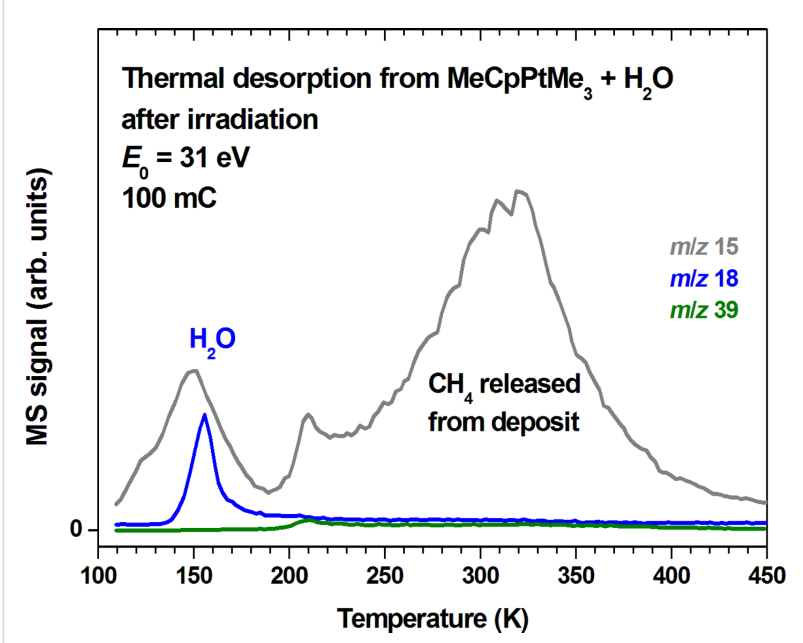

Figure 7: Thermal desorption spectra obtained after an electron exposure of $100 \mathrm{mC}$ at $E_{0}=31 \mathrm{eV}$ from a 15 layer film of $\mathrm{MeCpPtMe}_{3}$ condensed on a Ta substrate held at $105 \mathrm{~K}$ and covered by a threefold excess of $\mathrm{H}_{2} \mathrm{O}$. The ratios $\mathrm{m} / \mathrm{z} 18$ and $\mathrm{m} / \mathrm{z} 15$ were recorded to monitor desorption of remaining $\mathrm{H}_{2} \mathrm{O}$ as well as possible formation of $\mathrm{CH}_{4}$. The $\mathrm{m} / \mathrm{z}$ ratio $39\left(\mathrm{C}_{3} \mathrm{H}_{3}{ }^{+}\right)$is included as a characteristic signal of $\mathrm{MeCpPtMe}_{3}$ and of $\mathrm{MeCpH}$ that may possibly be released from the decomposed layer.

We note that desorption of $\mathrm{MeCpH}$ was not observed although proton transfer to the precursor is antipicated when $\mathrm{MeCpPtMe}_{3}$ is irradiated in the presence of $\mathrm{H}_{2} \mathrm{O}$. This is obvious from a lack of desorption signal below the precursor desorption temperature in the $m / z 39$ data shown in Figure 7. Unlike the CVD process in the presence of $\mathrm{H}_{2}$ where the ligands are converted to their fully reduced and thus volatile analogues [19], thermal reactions following electron exposure in the presence of $\mathrm{H}_{2} \mathrm{O}$ do not remove the cyclopentadienyl ligands from the deposit as such or in a reduced form. In fact, not only $\mathrm{MeCpH}$ but also its reduced analogues 1-methylcyclopentene and methylcyclopentane have a medium intense fragment with $\mathrm{m} / \mathrm{z} 39$ [32] and would be expected to desorb at a similar temperature as $\mathrm{MeCpH}$. In conclusion, the cyclopentadienyl ligands must have been further decomposed in the present experiments.

\section{Water- and electron-induced degradation of a FEBID-like deposit produced from $\mathrm{MeCpPtMe}_{3}$}

The FEBID deposits produced from $\mathrm{MeCpPtMe}_{3}$ and used in previous purification experiments by post-deposition electronbeam treatment in the presence of water vapour had thickness up to $300 \mathrm{~nm}$, of which the topmost $45 \mathrm{~nm}$ were fully purified [12]. Taking the effective diameter of $0.96 \mathrm{~nm}$ of an $\mathrm{MeCpPtMe}_{3}$ molecule [33] and considering that condensed precursor layers lose organic material and densify during degradation, a 30 monolayer condensed precursor layer would yield a deposit that is significantly thinner than such a typical FEBID deposit. To mimic a thick deposit, 30 monolayer films of $\mathrm{MeCpPtMe}_{3}$ were thus repeatedly deposited and exposed to electron irradiation at $E_{0}$ varying between $19 \mathrm{eV}$ and $31 \mathrm{eV}$ without intermittent sputter cleaning. Irradiation was performed for each deposition cycle until the formation of volatile $\mathrm{CH}_{4}$ had ceased. Remaining intact precursor molecules were removed from the surface by thermal annealing to $450 \mathrm{~K}$. The annealing step also initiates the thermal reactions that contribute to deposit formation in an actual FEBID process as obvious from our TDS data shown in Figure 4 and Figure 7. In total, more than 500 monolayers of the precursor were thus deposited on the surface so that the resulting deposit had an estimated thickness in the range of a typical FEBID nanostructure [12]. This deposit was then used for a desorption experiment to study the reactions occuring during a water-assisted purification process (Figure 8 ). We note that due to the necessity of cryogenic conditions imposed by our experiments, this process of deposit formation is not performed under the typical steadystate conditions of an electron-limited FEBID process. It is, however, similar in the sense that the precursor is replenished after each individual deposit formation cycle.

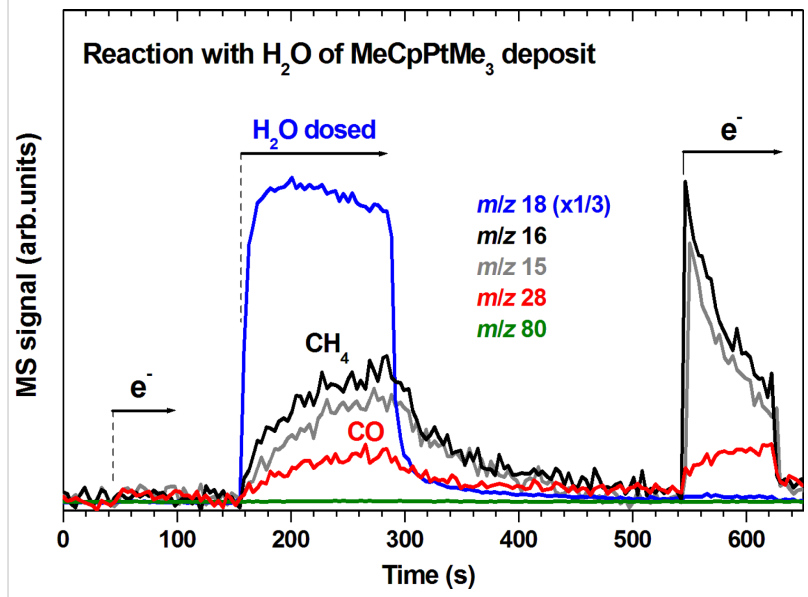

Figure 8: Desorption of $\mathrm{CH}_{4}(\mathrm{~m} / \mathrm{z} 15$ and 16$)$ and $\mathrm{CO}(\mathrm{m} / \mathrm{z} 28)$ from a deposit produced from more than 500 monolayers of $\mathrm{MeCpPtMe}_{3}$ deposited successively on a Ta substrate held at $108 \mathrm{~K}$ and decomposed by electron irradiation at $E_{0}$ varying between $19 \mathrm{eV}$ and $31 \mathrm{eV}$ followed in each deposition by annealing to $450 \mathrm{~K}$ to remove remaining intact precursor molecules. The $\mathrm{m} / \mathrm{z}$ ratio 18 serves to monitor $\mathrm{H}_{2} \mathrm{O}$ leaked into the chamber or possible desorption of $\mathrm{H}_{2} \mathrm{O}$ from the surface during subsequent electron exposure. The thick deposit was held at $108 \mathrm{~K}$ and first exposed to electron irradiation at $E_{0}=19 \mathrm{eV}$ $\left(1 \mathrm{mC} / \mathrm{cm}^{2}\right)$ (between $40 \mathrm{~s}$ and $100 \mathrm{~s}$ ) where lack of ESD gives evidence of complete precursor degradation. $\mathrm{H}_{2} \mathrm{O}$ was then leaked into the chamber (between $155 \mathrm{~s}$ and $280 \mathrm{~s}$ ) leading to surface reactions and consequent desorption of $\mathrm{CH}_{4}$ (identified from the characteristic intensity ratio for $\mathrm{m} / \mathrm{z} 16$ and $\mathrm{m} / \mathrm{z} 15$ of 1:0.9 [10]) and CO ( $\mathrm{m} / \mathrm{z} 28)$. Finally, the deposit was again irradiated at $E_{0}=19 \mathrm{eV}\left(1 \mathrm{mC} / \mathrm{cm}^{2}\right)$ between $545 \mathrm{~s}$ and $630 \mathrm{~s}$, now leading to $\mathrm{ESD}$ of $\mathrm{CH}_{4}$ and $\mathrm{CO}$. Included is also $\mathrm{m} / \mathrm{z} 80$ which is representative of $\mathrm{MeCpH}$ [32] thus ruling out any noticeable desorption of the MeCp ligand as a result of possible protonation. 
Prior to the start of the experimental period shown in Figure 8, the thick deposit has been subject to a few cycles of deposition of $\mathrm{H}_{2} \mathrm{O}$, electron exposure, and annealing to $450 \mathrm{~K}$. Figure 8 thus represents the chemistry during an ongoing deposit purification process. Following the previous treatment and shown as a first step in Figure 8, the lack of ESD from the precursor layer was verified by an electron irradiation period between $40 \mathrm{~s}$ and $100 \mathrm{~s}$. At this stage, volatile species that were formed during previous purification cycles had been thermally desorbed prior to electron exposure and the irradiation step clearly did not produce further volatile products. Between $155 \mathrm{~s}$ and $280 \mathrm{~s}$, vapour of $\mathrm{H}_{2} \mathrm{O}$ was leaked into the chamber and thus allowed to condense on or penetrate the deposit still held at $108 \mathrm{~K}$. This induced reactions in the deposit as obvious from the onset of desorption of $\mathrm{CH}_{4}$ and $\mathrm{CO}$ at $155 \mathrm{~s}$ and increasing desorption rate afterwards. This shows that, although the previous electron exposure has not removed organic material, it has nonetheless activated the deposit. The deposited material thus thermally reacted with $\mathrm{H}_{2} \mathrm{O}$ even at cryogenic temperature. The resulting reactions are more obvious from the thick deposit (Figure 8) than from a thin degraded layer (Figure 5 and Figure 6) most likely because the thick layer can maintain a larger number of activated species. Desorption of products decreased again when the supply of $\mathrm{H}_{2} \mathrm{O}$ vapour was stopped indicating again that $\mathrm{H}_{2} \mathrm{O}$ is the rate limiting factor under the given conditions. After desorption had completely ceased, a second electron irradiation cycle was applied to the deposit. This led to immediate desorption of more $\mathrm{CH}_{4}$ but subsequent decay (Figure 8) similar to the ESD of $\mathrm{CH}_{4}$ observed upon degradation of a fresh precursor layer (Figure 3). Further $\mathrm{CO}$ was also produced but, in contrast to $\mathrm{CH}_{4}$, its desorption rate increased during ongoing irradiation. This indicates again that $\mathrm{CO}$ was not trapped in the film as such but produced during electron exposure from an intermediate species.

Figure 8 shows clearly that thick FEBID like deposits release substantial amounts of $\mathrm{CH}_{4}$ and $\mathrm{CO}$ under irradiation in the presence of water. The results indicate that, following extensive electron exposure, a FEBID deposit produced from $\mathrm{MeCpPtMe}_{3}$ may still be reactive and thus change its composition when it later encounters vapour of $\mathrm{H}_{2} \mathrm{O}$. We note that the rate limiting effect of $\mathrm{H}_{2} \mathrm{O}$ was also visible during a later stage of the experiment where the vapour pressure of $\mathrm{H}_{2} \mathrm{O}$ above the deposit and thus the supply of $\mathrm{H}_{2} \mathrm{O}$ to the deposit was increased during electron exposure. This also led to an increase in product desorption rates (Supporting Information File 1, Figure S4).

It is well known that FEBID processes based on $\mathrm{MeCpPtMe}_{3}$ produce a nanogranular material consisting of Pt nanoparticles embedded in a carbonaceous matrix [12]. We note that the repeated cycles of applying $\mathrm{H}_{2} \mathrm{O}$ to the deposit followed by electron irradiation and annealing in fact has removed noticeable amounts of carbon. This was visible in AES data (Supporting Information File 1, Figure S5). While prior to purification, the signals of Pt embedded in the dominant amount of carbonaceous material were not visible, they clearly emerged after repeated purification cycles, underlining that these have in fact removed at least part of the carbon content in the present model experiments.

\section{Possible reaction pathways leading to formation of $\mathrm{CO}$}

The observed formation of $\mathrm{CO}$ as well as the degradation of the $\mathrm{MeCp}$ ligand that is obvious from the lack of $\mathrm{MeCpH}$ desorption in TDS experiments (Figure 7 and Figure 8) must both involve multiple bond dissociation or rearrangement. Catalytic reactions are one possibility to explain this result. A catalytic action of Pt in complexes resulting from electron-induced loss of ligands from $\mathrm{MeCpPtMe}_{3}$ provides a conceivable scenario for the formation of $\mathrm{CO}$ upon exposure of the deposit to $\mathrm{H}_{2} \mathrm{O}$ without electron irradiation. $\mathrm{Pt}(\mathrm{II})$ species are possibly formed when ligands are dissociated from the precursor. Such complexes are capable of inserting into $\mathrm{C}-\mathrm{H}$ bonds [34], a reaction that is fundamental to the known catalytic action of $\mathrm{Pt}$ for the oxidation of organic compounds in aqueous solution [30]. Such reactions might also contribute to the degradation of the $\mathrm{MeCp}$ anionic ligand and thus prevent its neutral form $\mathrm{MeCpH}$ from being detected. Also, catalytic reactions may proceed on the surface of Pt nanoparticles [22,23] as produced during deposit formation [12]. This idea is supported by the previous gas-phase study that included not only $\mathrm{PtCH}_{2}{ }^{+}$but also small clusters $\mathrm{Pt}_{\mathrm{n}} \mathrm{CH}_{2}{ }^{+}$and reported that small $\mathrm{Pt}_{\mathrm{n}}{ }^{+}$clusters have significantly higher activity with respect to oxidation of $\mathrm{CH}_{4}$ by $\mathrm{H}_{2} \mathrm{O}$ than $\mathrm{Pt}^{+}$[23]. Within this scenario, the more obvious release of volatile products without electron irradiation from the thick deposit (Figure 8) as compared to the decomposed thin multilayer film (Figure 5 and Figure 6) can be explained as an effect of a larger number of nanoparticles. However, detailed microscopic studies would be required to support this interpretation.

Alternatively, the formation of $\mathrm{CO}$ during electron exposure in the presence of $\mathrm{H}_{2} \mathrm{O}$ can be explained by an electron-driven reaction. In fact, this type of chemistry can explain both the degradation of the MeCp ligand as well as the formation of CO. As previously shown, $\mathrm{CO}$ is produced during electron-driven degradation of methanol which is the smallest alcohol [35]. Alcohols, on the other hand, can be formed through electroninitiated reactions. This was demonstrated for a condensed mixture of ethylene $\left(\mathrm{C}_{2} \mathrm{H}_{4}\right)$ and $\mathrm{H}_{2} \mathrm{O}$ [31]. Electron exposure initiated addition of $\mathrm{H}_{2} \mathrm{O}$ to the $\mathrm{CC}$ double bond of $\mathrm{C}_{2} \mathrm{H}_{4}$ yielding ethanol $\left(\mathrm{C}_{2} \mathrm{H}_{5} \mathrm{OH}\right)$. The reaction proceeds through both an ionization-driven reaction and a complex mechanism involving 
DEA [31]. This led us to the hypothesis that electron irradiation may also drive addition of $\mathrm{H}_{2} \mathrm{O}$ to the $\mathrm{MeCp}$ ligand of $\mathrm{MeCpPtMe}_{3}$ or to $\mathrm{MeCpH}$ released from the precursor upon electron-driven proton transfer (see above). The resulting alcohol would then further decompose to release CO.

Beside formation of an alcohol, other types of reactions are conceivable. Representative isomers of the different types of reaction products are listed in Table 1. In fact, electron-induced $\mathrm{CH}$ bond cleavage releases atomic hydrogen that, in a condensed phase, can add to $\mathrm{CC}$ double bonds. This can induce a sequence of reactions that leads to formation of the saturated analogue of an initially unsaturated hydrocarbon molecule [36]. In the case of $\mathrm{MeCpH}$, such a reduction of one of the two double bonds would lead to the different isomers of methylcyclopentene. Also, it is known that single electron oxidation of dienes significantly reduces the activation barrier towards formation of the Diels-Alder dimer [37] so that this product is also anticipated under electron exposure at $E_{0}$ above the ionization threshold. Table 1 also includes characteristic mass spectrometric signals of these different types of compounds as a basis for the analysis of our experimental product identification.

To investigate if an alcohol is indeed formed, a mixed condensed layer of methylcyclopentadiene $(\mathrm{MeCpH})$ and $\mathrm{H}_{2} \mathrm{O}$ was irradiated and subsequently analyzed by TDS. According to literature, the mass spectrum of the anticipated alcohol that would result from addition of $\mathrm{H}_{2} \mathrm{O}$ to $\mathrm{MeCpH}$, represented by 1-methyl-2-cyclopenten-1-ol is dominated by a signal at $\mathrm{m} / \mathrm{z} 83$ [38]. Other signals near and above this mass were small while signals below $\mathrm{m} / \mathrm{z} 79$ were not reported. Figure 9 shows TDS scans recorded after electron exposure to the mixed layer of $\mathrm{MeCpH}$ and $\mathrm{H}_{2} \mathrm{O}$ including the anticipated $\mathrm{m} / \mathrm{z}$ 83. In fact, a desorption signal with maximum around $190 \mathrm{~K}$ is seen in the TDS scan at $m / z 83$ as well as in scans recorded at $m / z 55$, and $m / z 43$. We note that the experiment was repeated several times to screen all conceivable mass fragments but only those that showed a desorption signal that apparently relates to the antici-

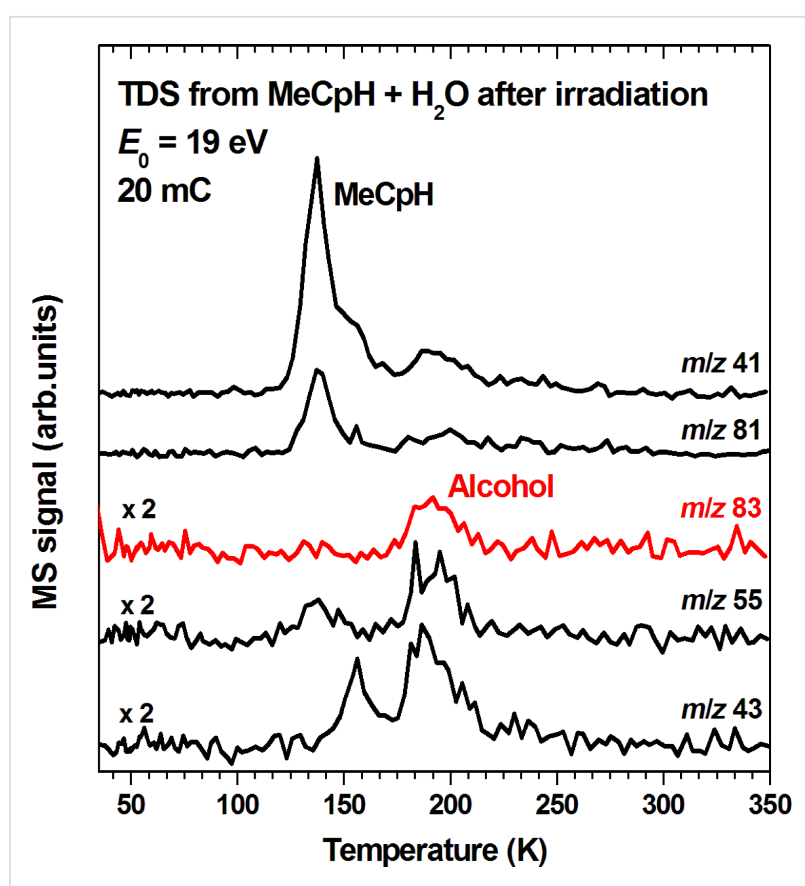

Figure 9: Thermal desorption spectra from a mixed condensed layer of $\mathrm{MeCpH}$ and $\mathrm{H}_{2} \mathrm{O}(1: 1)$ with estimated thickness corresponding to 20 monolayers on a Au substrate recorded after an electron exposure of $4 \mathrm{mC} / \mathrm{cm}^{2}$ at $E_{0}=19 \mathrm{eV}$ with the substrate held at $35 \mathrm{~K}$. See text and Table 1 for explanations on selection of $\mathrm{m} / \mathrm{z}$ ratios shown here.

pated product are shown here. $\mathrm{m} / \mathrm{z} 41$ and $\mathrm{m} / \mathrm{z} 81$ are included because they are minor fragments in the EI mass spectrum of $\mathrm{MeCpH} \mathrm{[32]} \mathrm{as} \mathrm{confirmed} \mathrm{by} \mathrm{the} \mathrm{characteristic} \mathrm{desorption}$ signal with maximum at $137 \mathrm{~K}$ (compare Supporting Information File 1, Figure S6).

To support the assignment of the $190 \mathrm{~K}$ desorption signal to the anticipated alcohol, other conceivable products must be ruled out. The mass spectra of all isomers of methylcyclopentene are dominated by a fragment with $m / z 67$ [32]. In fact, an $m / z 67$ desorption signal emerges at $135 \mathrm{~K}$ upon electron exposure of a pure condensed layer of $\mathrm{MeCpH}$ while $\mathrm{m} / \mathrm{z} 83$ is not observed under these conditions (Figure 10, top). This clearly shows that

Table 1: Anticipated products of electron-induced reactions of pure $\mathrm{MeCpH}$ or of $\mathrm{MeCpH}$ in presence of $\mathrm{H}_{2} \mathrm{O}$.

$\mathrm{MeCpH}$

Reaction

Electron-induced hydration [31] Reduction by atomic hydrogen resulting

Anticipated reaction products

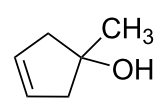

Characteristic MS $m / z 83\left[\mathrm{M}-\mathrm{CH}_{3}\right]^{\bullet+}[38]$ fragments from electron-induced $\mathrm{CH}$ bond cleavage of adjacent molecules [36]

Electron-induced dimer formation (Diels-Alder-type reaction) [37]

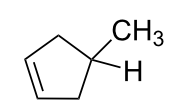

$m / z 67\left[\mathrm{M}-\mathrm{CH}_{3}\right]^{\bullet+}[32]$<smiles>CC1=CC2CC3(C)C=CC2C3C1</smiles>

$\mathrm{m} / \mathrm{z} 81$ [MeCpH$\left._{2}\right]^{+}$[this work] 
the $m / z 83$ desorption signal at $190 \mathrm{~K}$ is not due to reduced forms of $\mathrm{MeCpH}$. In contrast, both the product with one reduced double bond $(\mathrm{m} / z 67$ at $135 \mathrm{~K})$ and the desorption signal at $190 \mathrm{~K}$ in $\mathrm{m} / z 83$ and as well as $m / z 55$ are observed when $\mathrm{MeCpH}$ mixed with $\mathrm{H}_{2} \mathrm{O}$ is irradiated (Figure 10, bottom) showing that reduction of $\mathrm{MeCpH}$ is a concurrent reaction to addition of $\mathrm{H}_{2} \mathrm{O}$ yielding an alcohol. At the same time, the absence of a noticeable desorption signal in the $\mathrm{m} / z 69$ curve which is one of the most intense fragments of methyl cyclopentane [32] rules out the hydrogenation of both double bonds.

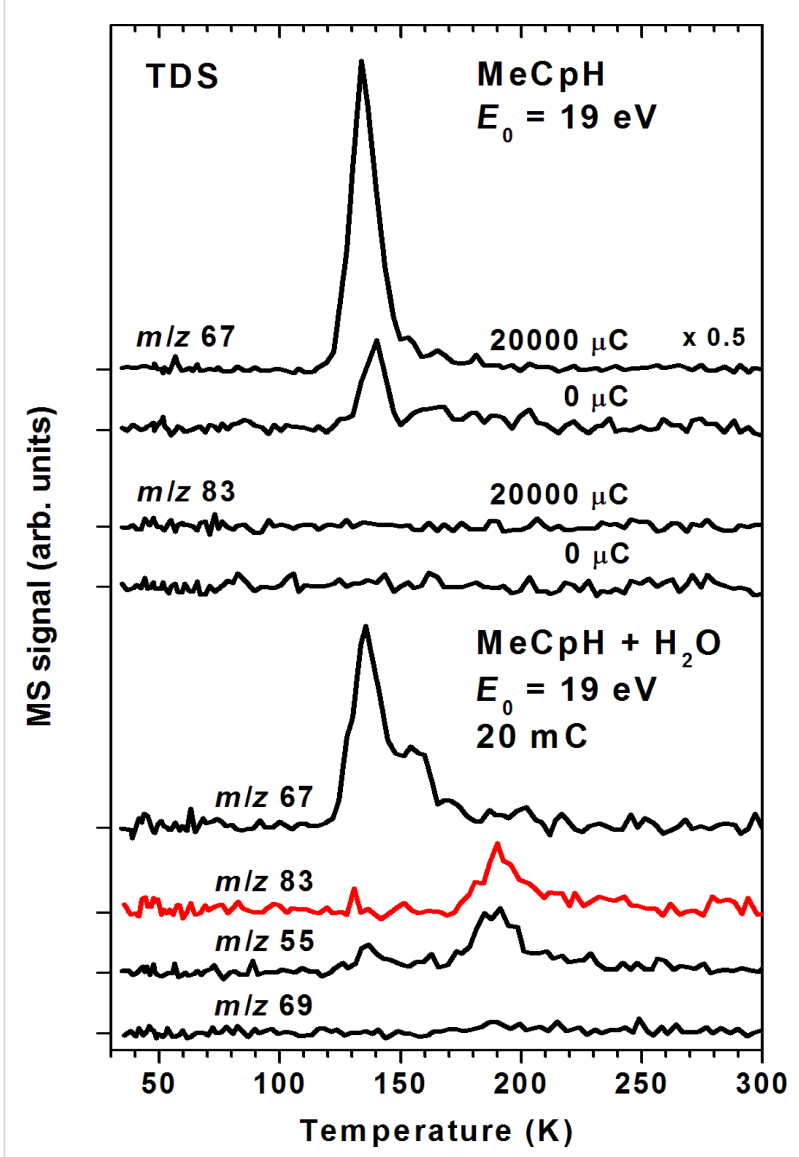

Figure 10: Thermal desorption spectra from condensed layers of $\mathrm{MeCpH}$ with estimated thickness corresponding to 15 monolayers on a Au substrate recorded before and after an electron exposure of $4 \mathrm{mC} / \mathrm{cm}^{2}$ at $E_{0}=19 \mathrm{eV}$ with the substrate held at $35 \mathrm{~K}$ (top) and of a mixed condensed layer of $\mathrm{MeCpH}$ and $\mathrm{H}_{2} \mathrm{O}$ (1:1) with estimated thickness corresponding to 20 monolayers on the same substrate recorded after an electron exposure of $4 \mathrm{mC} / \mathrm{cm}^{2}$ at $E_{0}=19 \mathrm{eV}$ with the substrate held at $35 \mathrm{~K}$ (bottom). See text and Table 1 for explanations on selection of $\mathrm{m} / \mathrm{z}$ ratios shown here.

While the methylcyclopentene isomers desorb at the same temperature as $\mathrm{MeCpH}$ itself, the higher desorption temperature of $190 \mathrm{~K}$ in the $\mathrm{m} / \mathrm{z} 83$ curve points to the formation of a larger product. However, a mass spectrum of the $\mathrm{MeCpH}$ dimer that was recorded as reference does not show a signal at $\mathrm{m} / \mathrm{z} 83$ but a medium intensity fragment at $\mathrm{m} / \mathrm{z} 81$. Such a significantly more intense $m / z 81$ signal is not seen around $190 \mathrm{~K}$ (see Figure 9). These arguments rule out an assignment of the $190 \mathrm{~K}$ desorption peak to the $\mathrm{MeCpH}$ dimer and thus support that the anticipated alcohol is in fact produced under electron exposure of $\mathrm{MeCpH}$ in the presence of $\mathrm{H}_{2} \mathrm{O}$.

The results show that addition of $\mathrm{H}_{2} \mathrm{O}$ to unsaturated hydrocarbon species in the deposit likely leads to alcoholic species which are likely to release $\mathrm{CO}$ during electron exposure [35]. To show that electron-induced reactions with $\mathrm{H}_{2} \mathrm{O}$ in fact convert carbon to $\mathrm{CO}$ in a deposit produced from $\mathrm{MeCpH}$, a large amount of the compound was slowly dosed onto the Ta substrate held at $105 \mathrm{~K}$. During dosing, the condensing layers were continuously irradiated with electrons at $E_{0}=31 \mathrm{eV}$ to build up a crosslinked carbonaceous layer. Remaining $\mathrm{MeCpH}$ was then removed by annealing to room temperature. After cooling down again, as shown in Figure 11, a first ESD experiment was performed leading to negligible production of volatile species. After irradiation was stopped, $\mathrm{H}_{2} \mathrm{O}$ was dosed onto the deposit yielding again no significant amounts of volatile products. However, a second period of electron exposure (between $540 \mathrm{~s}$ and $660 \mathrm{~s}$ ) led not only to immediate desorption of $\mathrm{H}_{2} \mathrm{O}$ that had condensed on or diffused into the deposit. Also,

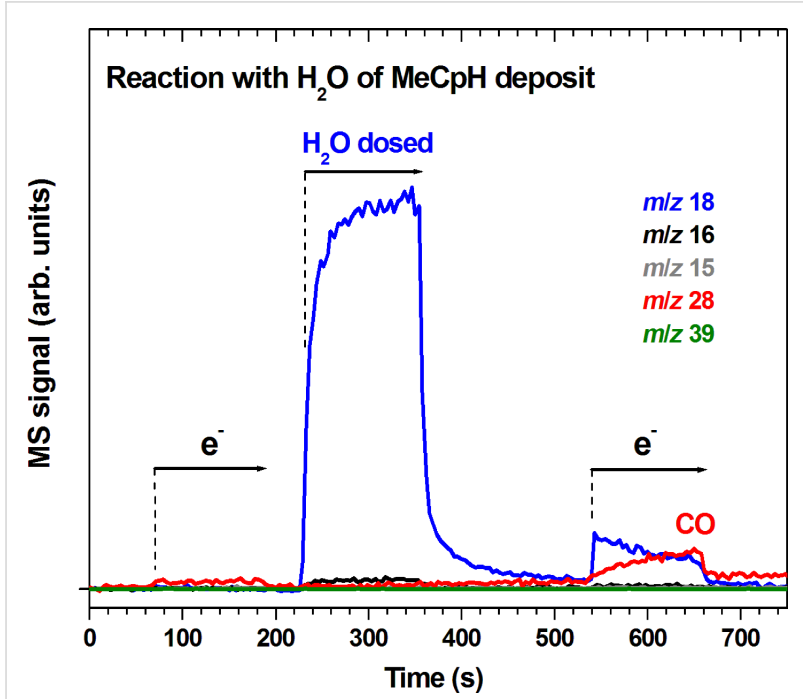

Figure 11: MS signals recorded during electron exposures $\left(\mathrm{e}^{-}\right)$and leaking of $\mathrm{H}_{2} \mathrm{O}\left(\mathrm{H}_{2} \mathrm{O}\right)$ onto a deposit that has been prepared by dosing a large amount of $\mathrm{MeCpH}$ onto a Ta substrate held at $105 \mathrm{~K}$ and simultaneous electron exposure at $E_{0}=31 \mathrm{eV}$ to build up a crosslinked carbonaceous layer. Remaining $\mathrm{MeCpH}$ was removed by annealing to room temperature prior to cooling the substrate down again and starting the experimental sequence shown here. During leaking of $\mathrm{H}_{2} \mathrm{O}$, an amount of vapour corresponding to 2-3 monolayers was applied. During the two electron irradiation periods, electron exposures of $2 \mathrm{mC} / \mathrm{cm}^{2}$ each were applied at $I_{\mathrm{p}}=17 \mu \mathrm{A} / \mathrm{cm}^{2}$ at $E_{0}=31 \mathrm{eV}$ The $m / z$ ratios $18,16,15$, and 28 were recorded to monitor leaking of $\mathrm{H}_{2} \mathrm{O}$ as well as possible formation of $\mathrm{CH}_{4}$ and $\mathrm{CO}$. The $\mathrm{m} / \mathrm{z}$ ratio 39 was included to show that desorption of $\mathrm{MeCpH}$ is negligible. 
desorption of $\mathrm{CO}$ was observed with increasing intensity during irradiation giving again evidence that an intermediate was formed that decomposed under electron exposure to yield $\mathrm{CO}$. It is very likely that addition of $\mathrm{H}_{2} \mathrm{O}$ to unsaturated molecular groups in the carbonaceous material resulting in formation of alcoholic species is also involved in this case. Such reactions can thus make contributions to the removal of carbon during deposit purification processes assisted by $\mathrm{H}_{2} \mathrm{O}$.

\section{Conclusion}

The present study uses a combination of different desorption experiments to obtain a deeper insight in the chemistry underlying the recently reported water-assisted purification of FEBID deposits produced from $\mathrm{MeCpPtMe}_{3}$ [12]. This approach combines thermal and electron-induced desorption spectrometry with experiments that monitor volatile products being formed upon contact of deposited material with vapour of $\mathrm{H}_{2} \mathrm{O}$. The experiments thus differentiate between electron-induced reactions and contributions of thermal chemistry to the formation of volatile decomposition products during precursor degradation. A comparison of MS intensities obtained during electron-stimulated desorption (ESD) and in a post-irradiation thermal desorption spectrometric (TDS) experiment allows us to estimate that more $\mathrm{CH}_{4}$ is released upon temperature increase than during irradiation of the precursor at liquid nitrogen temperature. These thermal processes must be taken into account when comparing the results of a surface science model study to an actual FEBID process.

Based on the present results in connection with previous experiments we can safely confirm that $\mathrm{CH}_{4}$ and not $\mathrm{CH}_{3}$ is released from the condensed layer of $\mathrm{MeCpPtMe}_{3}$ during electron-induced fragmentation. This finding suggests that efficient reaction channels exist in the condensed layer that either produce $\mathrm{CH}_{4}$ or retain $\mathrm{CH}_{3}$ in the deposit.

Regarding the water-assisted purification of FEBID deposits produced from $\mathrm{MeCpPtMe}_{3}$, the present experiments show that the combined action of $\mathrm{H}_{2} \mathrm{O}$ and electron exposure is required for an efficient removal of organic material at cryogenic temperature. However, evidence was obtained that the reaction rates are limited by the available supply of $\mathrm{H}_{2} \mathrm{O}$ under the given vacuum conditions. We propose that ionization of $\mathrm{H}_{2} \mathrm{O}$ and subsequent proton transfer and formation of $\mathrm{OH}$ radicals is an essential reaction step in deposit purification chemistry. The fact that $\mathrm{OH}$ radicals are involved can also rationalize the depth dependence of the efficiency of electron-driven deposit purification processes assisted by $\mathrm{H}_{2} \mathrm{O}$ as reported previously [12].

Desorption of $\mathrm{CO}$ that is produced beside $\mathrm{CH}_{4}$ during the waterassisted purification reactions is typically delayed as compared to $\mathrm{CH}_{4}$. This implies that $\mathrm{CO}$ is released from an intermediate product. We propose that electron-induced reactions lead to alcoholic species as intermediates. These are formed by addition of $\mathrm{H}_{2} \mathrm{O}$ to unsaturated molecular groups in the carbonaceous material resulting from decomposition of the precursor ligands. Further electron-induced decomposition of this alcohol then yields CO. This also accounts for the finding that the cyclopentadienyl ligand cannot be desorbed from the deposit as an intact unit. We propose that such reactions are relevant to the removal of carbon during deposit purification processes assisted by $\mathrm{H}_{2} \mathrm{O}$ and also to the recently reported electron-induced etching of graphene using an ice layer as resist [17]. Contributions from catalytic chemistry on Pt complexes and/or Pt nanoparticles are also conceivable. However, further studies using, in particular, quantum chemistry are needed to gain more insight in such reactions as well as in the properties of reactive intermediates involved in precursor decomposition and deposit purification processes.

Finally, the present results also suggest that under conditions relevant to the actual technical process, FEBID deposits may react with $\mathrm{H}_{2} \mathrm{O}$ even in the absence of electron irradiation. Specific attention should thus be paid to stability under moist conditions of devices fabricated by FEBID from $\mathrm{MeCpPtMe}_{3}$.

\section{Experimental}

All experiments were performed in an ultrahigh vacuum (UHV) chamber [39] with a base pressure of about $10^{-10}$ mbar. In all experiments, multilayer films of $\mathrm{MeCpPtMe}_{3}$ were condensed on a polycrystalline Ta sheet held between $105 \mathrm{~K}$ and $110 \mathrm{~K}$ by liquid $\mathrm{N}_{2}$ cooling. The condition of the Ta substrate was monitored by Auger electron spectroscopy (STAIB DESA 100). Prior to an experiment, the substrate was sputter-cleaned using $\mathrm{Ar}^{+}$ions at $3 \mathrm{keV}$ until the Auger signals of the underlying $\mathrm{Ta}$ were clearly visible. Immediately before the precursor deposition, adsorbed volatile compounds from the residual gas were further removed by annealing to $450 \mathrm{~K}$ through resistive heating of two thin Ta ribbons spot-welded to the thicker Ta sheet.

To produce the precursor films, vapours of $\mathrm{MeCpPtMe}_{3}$ were introduced via a gas handling manifold consisting of precision leak valves and a small calibrated volume where the absolute pressure is measured with a capacitance manometer. For each film deposition, a calibrated amount of vapour was leaked via a stainless steel capillary opening onto the metal substrate. Experiments on methylcyclopentadiene $(\mathrm{MeCpH})$ were performed on a polycrystalline $\mathrm{Au}$ sheet held at $35-38 \mathrm{~K}$ by a closed-cycle helium refrigerator (Leybold Vacuum). This substrate was cleaned between the experiments by annealing to $400 \mathrm{~K}$ again using two thin Ta resistive heating ribbons spot-welded to the thicker Au sheet. 
The film thickness of $\mathrm{MeCpPtMe}_{3}$ layers was estimated by thermal desorption spectrometry (TDS) of films with increasing coverage. The data recorded at $16 \mathrm{amu}$ (Supporting Information File 1, Figure S7) show a broad and weak desorption signal with maximum around $230 \mathrm{~K}$ which rapidly saturates and is therefore ascribed to the monolayer. A second peak with maximum at $210 \mathrm{~K}$ starts to increase upon saturation of the monolayer peak and is hence attributed to the successive layers no longer in contact with the substrate. The multilayer desorption temperature agrees with values observed previously on $\mathrm{Au}(110)$ [10]. In the same way, the monolayer coverage of $\mathrm{MeCpH}$ was estimated (Supporting Information File 1, Figure S6). In all experiments, multilayer films with a thickness of 30 layers $( \pm 30 \%)$ were prepared. In purification and control experiments, typical quantities of $\mathrm{H}_{2} \mathrm{O}$ vapour leaked into the chamber corresponded to those used for deposition of $\mathrm{MeCpPtMe}_{3}$ and $\mathrm{MeCpH}$ (see also Figure captions).

Desorption experiments were performed by use of a quadrupole mass spectrometer (QMS) residual gas analyser (Stanford, $300 \mathrm{amu}$ ) with electron impact ionization at $70 \mathrm{eV}$. The sample temperature was measured using a type $\mathrm{E}$ thermocouple pressfitted to the Ta or Au substrate. For electron-stimulated desorption (ESD) isothermal experiments, the sample was kept at the lowest attainable temperature and exposed to electron irradiation using a commercial flood gun (SPECS FG 15/40). This electron source delivers electrons with tunable kinetic energy $\left(E_{0}\right)$ at an estimated resolution of the order of $0.5-1 \mathrm{eV}$ and currents as measured at the substrate $\left(I_{\mathrm{p}}\right)$ of the order of up to $150 \mu \mathrm{A}$ for an irradiated area of $5 \mathrm{~cm}^{2}$. Desorption of volatile species was also monitored, again under isothermal conditions, upon dosing of $\mathrm{H}_{2} \mathrm{O}$, either without or with simultaneous electron exposure. Thermal desorption spectrometry (TDS) was performed by applying a temperature ramp of $1 \mathrm{~K} / \mathrm{s}$ to the sample.

$\mathrm{MeCpPtMe}_{3}$ was purchased from STREM and ACROS, both at a stated purity of $99 \%$, and degassed by repeated freeze-pumpthaw cycles. $\mathrm{MeCpH}$ was a mixture of isomers (1-, 2-, and 3 -methylcyclopenta-1,3-diene) and prepared from the dimer by thermal cracking above $172{ }^{\circ} \mathrm{C}$. It was kept below $-20{ }^{\circ} \mathrm{C}$ in the sample reservoir throughout the experiments to prevent the reverse reaction to the dimer.

\section{Supporting Information}

\section{Supporting Information File 1}

Additional experimental data.

[http://www.beilstein-journals.org/bjnano/content/

supplementary/2190-4286-9-10-S1.pdf]

\section{Acknowledgements}

This work was conducted within the framework of the COST Action CM1301 (CELINA).

\section{ORCID ${ }^{\circledR}$ iDs}

Markus Rohdenburg - https://orcid.org/0000-0001-8533-3610

Petra Swiderek - https://orcid.org/0000-0002-4355-5546

\section{References}

1. Utke, I.; Hoffmann, P.; Melngailis, J. J. Vac. Sci. Technol., B 2008, 26, 1197-1276. doi:10.1116/1.2955728

2. Noh, J. H.; Stanford, M. G.; Lewis, B. B.; Fowlkes, J. D.; Plank, H.; Rack, P. D. Appl. Phys. A 2014, 117, 1705-1713. doi:10.1007/s00339-014-8745-0

3. Utke, I.; Gölzhäuser, A. Angew. Chem., Int. Ed. 2010, 49, 9328-9330. doi:10.1002/anie.201002677

4. Höflich, K.; Yang, R. B.; Berger, A.; Leuchs, G.; Christiansen, S. Adv. Mater. 2011, 23, 2657-2661. doi:10.1002/adma.201004114

5. Winkler, R.; Schmidt, F.-P.; Haselmann, U.; Fowlkes, J. D.; Lewis, B. B.; Kothleitner, G.; Rack, P. D.; Plank, H. ACS Appl. Mater. Interfaces 2017, 9, 8233-8240. doi:10.1021/acsami.6b13062

6. Huth, M.; Porrati, F.; Schwalb, C.; Winhold, M.; Sachser, R.; Dukic, M.; Adams, J.; Fantner, G. Beilstein J. Nanotechnol. 2012, 3, 597-619. doi:10.3762/bjnano.3.70

7. Pablo-Navarro, J.; Sanz-Hernández, D.; Magén, C.; Fernández-Pacheco, A.; de Teresa, J. M. J. Phys. D: Appl. Phys. 2017, 50, 18LT01. doi:10.1088/1361-6463/aa63b4

8. Pohlit, M.; Eibisch, P.; Akbari, M.; Porrati, F.; Huth, M.; Müller, J. Rev. Sci. Instrum. 2016, 87, 113907. doi:10.1063/1.4967940

9. Botman, A.; Mulders, J. J. L.; Hagen, C. W. Nanotechnology 2009, 20, 372001. doi:10.1088/0957-4484/20/37/372001

10. Wnuk, J. D.; Gorham, J. M.; Rosenberg, S.; van Dorp, W. F.; Madey, T. E.; Hagen, C. W.; Fairbrother, D. H. J. Phys. Chem. C 2009, 113, 2487-2496. doi:10.1021/jp807824c

11. Thorman, R. M.; Kumar T. P., R.; Fairbrother, D. H.; Ingólfsson, O. Beilstein J. Nanotechnol. 2015, 6, 1904-1926. doi:10.3762/bjnano.6.194

12. Geier, B.; Gspan, C.; Winkler, R.; Schmied, R.; Fowlkes, J. D.; Fitzek, H.; Rauch, S.; Rattenberger, J.; Rack, P. D.; Plank, H. J. Phys. Chem. C 2014, 118, 14009-14016. doi:10.1021/jp503442b

13. Kolb, F.; Schmoltner, K.; Huth, M.; Hohenau, A.; Krenn, J.; Klug, A.; List, E. J. W.; Plank, H. Nanotechnology 2013, 24, 305501. doi:10.1088/0957-4484/24/30/305501

14. Mehendale, S.; Mulders, J. J. L.; Trompenaars, P. H. F. Nanotechnology 2013, 24, 145303. doi:10.1088/0957-4484/24/14/145303

15. Villamor, E.; Casanova, F.; Trompenaars, P. H. F.; Mulders, J. J. L. Nanotechnology 2015, 26, 095303. doi:10.1088/0957-4484/26/9/095303

16. Plank, H.; Noh, J. H.; Fowlkes, J. D.; Lester, K.; Lewis, B. B.; Rack, P. D. ACS Appl. Mater. Interfaces 2014, 6, 1018-1024. doi:10.1021/am4045458

17. Gardener, J. A.; Golovchenko, J. A. Nanotechnology 2012, 23, 185302 doi:10.1088/0957-4484/23/18/185302 
18. Setthapun, W.; Williams, W. D.; Kim, S. M.; Feng, H.; Elam, J. W.; Rabuffetti, F. A.; Poeppelmeier, K. R.; Stair, P. C.; Stach, E. A.; Ribeiro, F. H.; Miller, J. T.; Marshall, C. L. J. Phys. Chem. C 2010, 114, 9758-9771. doi:10.1021/jp911178m

19. Xue, Z.; Thridandam, H.; Kaesz, H. D.; Hicks, R. F. Chem. Mater. 1992, 4, 162-166. doi:10.1021/cm00019a032

20. Engmann, S.; Stano, M.; Matejčík, Š.; Ingólfsson, O. Phys. Chem. Chem. Phys. 2012, 14, 14611-14618. doi:10.1039/c2cp42637d

21. Warneke, J.; Van Dorp, W. F.; Rudolf, P.; Stano, M.; Papp, P.; Matejčí, Š.; Borrmann, T.; Swiderek, P. Phys. Chem. Chem. Phys. 2015, 17, 1204-1216. doi:10.1039/C4CP04239E

22. Mackus, A. J. M.; Leick, N.; Baker, L.; Kessels, W. M. M. Chem. Mater. 2012, 24, 1752-1761. doi:10.1021/cm203812v

23. Koszinowski, K.; Schröder, D.; Schwarz, H. Organometallics 2003, 22, 3809-3819. doi:10.1021/om030272l

24. Landheer, K.; Rosenberg, S. G.; Bernau, L.; Swiderek, P.; Utke, I.; Hagen, C. W.; Fairbrother, D. H. J. Phys. Chem. C 2011, 115, 17452-17463. doi:10.1021/jp204189k

25. Rosenberg, S. G.; Landheer, K.; Hagen, C. W.; Fairbrother, D. H. J. Vac. Sci. Technol., B 2012, 30, 051805. doi:10.1116/1.4751281

26. Hedhili, M. N.; Bredehöft, J. H.; Swiderek, P. J. Phys. Chem. C 2009, 113, 13282-13286. doi:10.1021/jp810834r

27. Garrett, B. C.; Dixon, D. A.; Camaioni, D. M.; Chipman, D. M.; Johnson, M. A.; Jonah, C. D.; Kimmel, G. A.; Miller, J. H.; Rescigno, T. N.; Rossky, P. J.; Xantheas, S. S.; Colson, S. D.; Laufer, A. H.; Ray, D.; Barbara, P. F.; Bartels, D. M.; Becker, K. H.; Bowen, K. H., Jr.; Bradforth, S. E.; Carmichael, I.; Coe, J. V.; Corrales, L. R.; Cowin, J. P.; Dupuis, M.; Eisenthal, K. B.; Franz, J. A.; Gutowski, M. S.; Jordan, K. D.; Kay, B. D.; LaVerne, J. A.; Lymar, S. V.; Madey, T. E.; McCurdy, C. W.; Meisel, D.; Mukamel, S.; Nilsson, A. R.; Orlando, T. M.; Petrik, N. G.; Pimblott, S. M.; Rustad, J. R.; Schenter, G. K.; Singer, S. J.; Tokmakoff, A.; Wang, L.-S.; Wittig, C.; Zwier, T. S. Chem. Rev. 2005, 105, 355-389. doi:10.1021/cr030453x

28. Maitlis, P. M. Chem. Soc. Rev. 1981, 10, 1-48. doi:10.1039/cs9811000001

29. Shaver, A. Can. J. Chem. 1978, 56, 2281-2285. doi:10.1139/v78-376

30. Labinger, J. A.; Herring, A. M.; Lyon, D. K.; Luinstra, G. A.; Bercaw, J. E.; Horvath, I. T.; Eller, K. Organometallics 1993, 12, 895-905. doi:10.1021/om00027a045

31. Warneke, J.; Wang, Z.; Swiderek, P.; Bredehöft, J. H. Angew. Chem., Int. Ed. 2015, 54, 4397-4400. doi:10.1002/anie.201412147

32. NIST Mass Spec Data Center. Mass Spectra. In NIST Chemistry WebBook, NIST Standard Reference Database Number 69; Linstrom, P. J.; Mallard, W. G., Eds.; National Institute of Standards and Technology: Gaithersburg MD, U.S.A., 2017. doi:10.18434/T4D303

33. Xue, Z.; Strouse, M. J.; Shuh, D. K.; Knobler, C. B.; Kaesz, H. D.; Hicks, R. F.; Williams, R. S. J. Am. Chem. Soc. 1989, 111, 8779-8784. doi:10.1021/ja00206a002

34. Lersch, M.; Tilset, M. Chem. Rev. 2005, 105, 2471-2526. doi:10.1021/cr030710y

35. Lepage, M.; Michaud, M.; Sanche, L. J. Chem. Phys. 1997, 107, 3478-3484. doi:10.1063/1.474485

36. Böhler, E.; Bredehöft, J. H.; Swiderek, P. J. Phys. Chem. C 2014, 118, 6922-6933. doi:10.1021/jp501192v

37. Schmittel, M.; Burghart, A. Angew. Chem., Int. Ed. Engl. 1997, 36, 2550-2589. doi:10.1002/anie.199725501
38. Chiu, I-C.; Kohn, H. J. Org. Chem. 1983, 48, 2857-2866. doi:10.1021/jo00165a015

39. Ipolyi, I.; Michaelis, W.; Swiderek, P. Phys. Chem. Chem. Phys. 2007, 9, 180-191. doi:10.1039/B612261B

\section{License and Terms}

This is an Open Access article under the terms of the Creative Commons Attribution License (http://creativecommons.org/licenses/by/4.0), which permits unrestricted use, distribution, and reproduction in any medium, provided the original work is properly cited.

The license is subject to the Beilstein Journal of Nanotechnology terms and conditions:

(http://www.beilstein-journals.org/bjnano)

The definitive version of this article is the electronic one which can be found at: doi:10.3762/bjnano.9.10 